ESAIM: M2AN 50 (2016) 237-261

DOI: $10.1051 / \mathrm{m} 2 \mathrm{an} / 2015041$
ESAIM: Mathematical Modelling and Numerical Analysis

www.esaim-m2an.org

\title{
OPTIMAL CONTROL OF THE FULL TIME-DEPENDENT MAXWELL EQUATIONS
}

\author{
VERA BOMMER $^{1}$ AND IRWIN YOUSEPT $^{1}$
}

\begin{abstract}
This paper analyzes the optimal control of the full time-dependent Maxwell equations. Our goal is to find an optimal current density and its time-dependent amplitude which steer the electric and magnetic fields to the desired ones. The main difficulty of the optimal control problem arises from the complexity of the Maxwell equations, featuring a first-order hyperbolic structure. We present a rigorous mathematical analysis for the optimal control problem. Here, the semigroup theory and the Helmholtz decomposition theory are the key tools in the analysis. Our theoretical findings include existence, strong regularity, and KKT theory. The corresponding optimality system consists of forward-backward Maxwell equations for the optimal electromagnetic and adjoint fields, magnetostatic saddle point equations for the optimal current density, and a projection formula for the optimal timedependent amplitude. A semismooth Newton algorithm in a function space is established for solving the nonlinear and nonsmooth optimality system. The paper is concluded by numerical results, where mixed finite elements and Crank-Nicholson schema are used.
\end{abstract}

Mathematics Subject Classification. 78A25, 35Q61, 49K20.

Received January 5, 2015. Revised April 24, 2015.

Published online January 14, 2016.

\section{INTRODUCTION}

Electromagnetics plays an important role in many modern applications and technologies. They include applications in the energy science, nanotechnology, life science, magnetic confinement fusion, magnetic levitation technology, microwave heating, sensor technology, and many more. The optimal control of such complex electromagnetic processes is challenging and requires a careful mathematical and numerical investigation. Let $\Omega \subset \mathbb{R}^{3}$ be a bounded domain with a connected Lipschitz boundary $\Gamma:=\partial \Omega$. Furthermore, we consider a control domain $\Omega_{c} \subseteq \Omega$ with a connected Lipschitz boundary $\Gamma_{c}:=\partial \Omega_{c}$. Our goal is to find an optimal current density in $\Omega_{c}$ and its time-dependent amplitude, which drive the electric and magnetic fields to the desired ones. More precisely,

\footnotetext{
Keywords and phrases. Optimal control, time-dependent Maxwell's equations, strongly continuous semigroup, Helmholtz decomposition, semismooth Newton.

1 Universität Duisburg-Essen, Fakultät für Mathematik, Thea-Leymann-Str. 9, 45127 Essen, Germany.

vera.bommer@uni-due.de; irwin.yousept@uni-due.de
} 
we consider the following optimal control problem:

$$
\begin{array}{r}
\text { Minimize } \frac{1}{2} \| \boldsymbol{E}(T) \\
-\boldsymbol{E}_{d}\left\|_{\boldsymbol{L}_{\epsilon}^{2}(\Omega)}^{2}+\frac{1}{2}\right\| \boldsymbol{H}(T)-\boldsymbol{H}_{d} \|_{\boldsymbol{L}_{\boldsymbol{\mu}}^{2}(\Omega)}^{2} \\
+\frac{\lambda_{\boldsymbol{u}}}{2}\|\operatorname{curl} \boldsymbol{u}\|_{\boldsymbol{L}^{2}\left(\Omega_{c}\right)}^{2}+\frac{\lambda_{a}}{2}\|a\|_{L^{2}(0, T)}^{2},
\end{array}
$$

subject to the full time-dependent Maxwell equations:

$$
\begin{cases}\epsilon \boldsymbol{E}_{t}-\operatorname{curl} \boldsymbol{H}+\sigma \boldsymbol{E}=\chi_{\Omega_{c}} \boldsymbol{u} a & \text { in } \Omega \times(0, T), \\ \mu \boldsymbol{H}_{t}+\operatorname{curl} \boldsymbol{E}=0 & \text { in } \Omega \times(0, T), \\ \boldsymbol{E} \times \boldsymbol{n}=0 & \text { in } \Gamma \times(0, T), \\ \boldsymbol{E}(\cdot, 0)=\boldsymbol{E}_{0} & \text { in } \Omega, \\ \boldsymbol{H}(\cdot, 0)=\boldsymbol{H}_{0} & \text { in } \Omega,\end{cases}
$$

and the divergence-free constraint (charge conservation law) on the current density $\boldsymbol{u}=\boldsymbol{u}(x)$ as well as pointwise box constraints on the time-dependent amplitude $a=a(t)$ :

$$
\left\{\begin{array}{l}
\operatorname{div} \boldsymbol{u}=0 \quad \text { in } \Omega_{c}, \\
a_{\min } \leq a(t) \leq a_{\max }
\end{array} \quad \text { a.e. in }(0, T) .\right.
$$

Here, $\boldsymbol{E}$ and $\boldsymbol{H}$ are the electric and magnetic fields. The matrix-valued coefficient functions $\epsilon, \mu, \sigma: \Omega \rightarrow$ $\mathbb{R}^{3 \times 3}$ denote, respectively, the electric permittivity, the magnetic permeability, and the electric conductivity. Furthermore, $\boldsymbol{n}$ is the unit outward normal to $\Gamma$. Analogously, we denote by $\boldsymbol{n}_{c}$ the unit outward normal to $\Gamma_{c}$. The vector fields $\boldsymbol{E}_{d}$ and $\boldsymbol{H}_{d}$ denote the desired electric and magnetic fields at the final time $T \in \mathbb{R}^{+}$ of the electromagnetic process. Moreover, the constants $a_{\min } \leq a_{\max }$ represent the minimum and maximum amplitudes allowed for the process, and $\lambda_{\boldsymbol{u}}, \lambda_{a} \in \mathbb{R}^{+}$are the control cost parameters. Note that $a_{\min }=a_{\max }$ is allowed. Therefore, the case with only one control variable $\boldsymbol{u}$ is also covered here. The precise mathematical assumption on the data involved in $(\mathrm{P})$ will be specified in next section.

The theory of the time-dependent Maxwell equations (1.1) requires the use of a weighted $\boldsymbol{L}^{2}$-Hilbert space, with two weight matrix functions given by the electric permittivity $\epsilon$ and the magnetic permeability $\mu$. This is the reason for considering the weighted norms $\|\cdot\|_{\boldsymbol{L}_{\epsilon}^{2}(\Omega)}^{2}:=(\epsilon \cdot, \cdot)_{\boldsymbol{L}^{2}(\Omega)}$ and $\|\cdot\|_{\boldsymbol{L}_{\mu}^{2}(\Omega)}^{2}:=(\mu \cdot, \cdot)_{\boldsymbol{L}^{2}(\Omega)}$ in the objective functional of $(\mathrm{P})$. Neglecting these weight matrix functions would then lead to an undesired low regularity property of the adjoint fields (see Rem. 3.5).

We employ $\boldsymbol{H}_{0}\left(\mathbf{c u r l} ; \Omega_{c}\right) \cap \boldsymbol{H}\left(\operatorname{div}=0 ; \Omega_{c}\right)$ for the feasible set of the current density $\boldsymbol{u}$ and include a Tikhonov-regularization term $\frac{\lambda_{u}}{2}\|\operatorname{curl} \boldsymbol{u}\|_{\boldsymbol{L}^{2}\left(\Omega_{c}\right)}^{2}$ in the objective functional of (P). In the case of time-dependent (parabolic) eddy current equations and magnetostatic Maxwell's equations (see [13], Sect. 2.3 and [34], Thm. 4.3), this term is not required, and the curl-regularity in the optimal current is a consequence of the optimality system. This is however not the case when dealing with the full Maxwell's equations (1.1). We underline that the curl-regularity in the current density $\boldsymbol{u}$ is required in order to prove that the optimal electromagnetic fields satisfy the Maxwell's equations (1.1) in the classical sense. Without the curl-regularity in the control $\boldsymbol{u}$, it is still possible to develop an optimal control theory in the mild (weak) sense (see the definition of mild solutions in (3.5)). However, the mild solution of (1.1) possesses only a low regularity property and does not even belong to the space $\boldsymbol{H}$ (curl). This would make the numerical computation considerably difficult, as the curl-regularity in the state is required in finite element method.

The optimal control problem (P) is mainly complicated by the complexity of the full time-dependent Maxwell equations (1.1) featuring a first-order hyperbolic coupled structure. Here, the key tools for the analysis of (P) are the semigroup theory and the Helmholtz decomposition theory. Our theoretical findings include existence, strong regularity result, and KKT theory for $(\mathrm{P})$. The corresponding optimality conditions are given by a coupled nonlinear system consisting of:

(i) Maxwell's equations for the optimal electromagnetic fields; 
(ii) backward Maxwell's equations for the adjoint fields;

(iii) magnetostatic saddle point equations for the optimal current density;

(iv) a projection formula for the optimal time-dependent amplitude.

The coupled nonlinear optimality system is reformulated as a nonsmooth operator equation, which we solve using a semismooth Newton method in function spaces. In every step of the algorithm, we employ the mixed method by [18] for the discretization of the forward-backward Maxwell system involving the lowest order edge elements of Nédélec's first family [20] and piecewise constant elements. Also, the discretization of the magnetostatic saddle point equations is based on a mixed finite element ansatz using the lowest order edge elements of Nédélec's first family and continuous piecewise linear elements.

To our knowledge, this paper is the first contribution to the optimal control of the full time-dependent Maxwell equations (P). In literature, we are only aware of the recent results for the optimal control of parabolic eddy current equations $[13,14,21,22]$, which are related to application problems in electromagnetic flow measurement (see also [11]). Earlier contributions to the boundary controllability of Maxwell's equations can be found in $[15,29]$. We also mention $[8,12,26,30-33]$ for the optimal control of linear time-harmonic eddy current equations (cf. [1]), whereas the nonlinear case has been recently investigated in [34]. The remainder of the paper is organized as follows. In next section, we introduce our notation and present all the function spaces and assumptions required for the analysis. Section 3 is devoted to the mathematical analysis of the optimal control problem, and a semismooth Newton algorithm is derived in Section 4. Numerical results are presented in the final section.

\section{Preliminaries}

We start by introducing our notation and general assumptions. Throughout the paper, $c$ denotes a generic positive constant. If $Y$ is a Hilbert space, then we use the notation $\|\cdot\|_{Y}$ and $(\cdot, \cdot)_{Y}$ for a standard norm and a standard scalar product on $Y$. If $Y$ is continuously embedded in another linear normed space $Z$, then we write $Y \hookrightarrow Z$. We use a bold typeface to indicate a three-dimensional vector function or a Hilbert space of three-dimensional vector functions. The following Hilbert spaces are used throughout the paper:

$$
\begin{array}{ll}
\boldsymbol{H}(\operatorname{curl}) & :=\left\{\boldsymbol{q} \in \boldsymbol{L}^{2}(\Omega) \mid \operatorname{curl} \boldsymbol{q} \in \boldsymbol{L}^{2}(\Omega)\right\}, \\
\boldsymbol{H}_{0}(\operatorname{curl}) & :=\{\boldsymbol{q} \in \boldsymbol{H}(\operatorname{curl}) \mid \boldsymbol{q} \times \boldsymbol{n}=0 \text { on } \Gamma\}, \\
\boldsymbol{H}\left(\operatorname{curl} ; \Omega_{c}\right) & :=\left\{\boldsymbol{q} \in \boldsymbol{L}^{2}\left(\Omega_{c}\right) \mid \operatorname{curl} \boldsymbol{q} \in \boldsymbol{L}^{2}\left(\Omega_{c}\right)\right\}, \\
\boldsymbol{H}_{0}\left(\operatorname{curl} ; \Omega_{c}\right) & :=\left\{\boldsymbol{q} \in \boldsymbol{H}\left(\operatorname{curl} ; \Omega_{c}\right) \mid \boldsymbol{q} \times \boldsymbol{n}_{c}=0 \text { on } \Gamma_{c}\right\}, \\
\boldsymbol{H}\left(\operatorname{div} ; \Omega_{c}\right) & :=\left\{\boldsymbol{q} \in \boldsymbol{L}^{2}\left(\Omega_{c}\right) \mid \operatorname{div} \boldsymbol{q} \in L^{2}\left(\Omega_{c}\right)\right\}, \\
\boldsymbol{H}_{0}\left(\operatorname{div} ; \Omega_{c}\right) & :=\left\{\boldsymbol{q} \in \boldsymbol{H}\left(\operatorname{div} ; \Omega_{c}\right) \mid \boldsymbol{q} \cdot \boldsymbol{n}_{c}=0 \text { on } \Gamma_{c}\right\}, \\
\boldsymbol{H}\left(\operatorname{div}=0 ; \Omega_{c}\right) & :=\left\{\boldsymbol{q} \in \boldsymbol{H}\left(\operatorname{div} ; \Omega_{c}\right) \mid \operatorname{div} \boldsymbol{q}=0 \text { in } \Omega_{c}\right\},
\end{array}
$$

where the curl- and div-operators as well as the tangential and normal traces are understood in the sense of distributions (see [9], Sect. 2 or [19], Sect. 3.5). Let us now summarize all the mathematical assumptions we require in this paper:

Assumption 2.1. The matrix-valued coefficient functions $\epsilon, \mu, \sigma: \Omega \rightarrow \mathbb{R}^{3 \times 3}$ have entries in $L^{\infty}(\Omega)$. The electric permittivity $\epsilon$ and the magnetic permeability $\mu$ are symmetric and uniformly positive definite, whereas 
the electric conductivity $\sigma$ is positive semidefinite, i.e.,

$$
\begin{aligned}
& \exists c_{\epsilon}>0, \quad \forall \xi \in \mathbb{R}^{3}, \quad \xi^{T} \epsilon(x) \xi \geq c_{\epsilon}|\xi|^{2} \text { a.e. in } \Omega, \\
& \exists c_{\mu}>0, \forall \xi \in \mathbb{R}^{3}, \quad \xi^{T} \mu(x) \xi \geq c_{\mu}|\xi|^{2} \text { a.e. in } \Omega \text {, } \\
& \forall \xi \in \mathbb{R}^{3}, \quad \xi^{T} \sigma(x) \xi \geq 0 \quad \text { a.e. in } \Omega \text {. }
\end{aligned}
$$

In the control domain $\Omega_{c}$, the electric permittivity $\epsilon$ is assumed to satisfy

$$
\epsilon(x)=\epsilon_{c}(x) I_{d} \quad \forall x \in \bar{\Omega}_{c},
$$

where $I_{d} \in \mathbb{R}^{3 \times 3}$ is the identity matrix and $\epsilon_{c} \in \mathcal{C}^{0,1}\left(\bar{\Omega}_{c}\right)$ with

$$
0<\underline{\epsilon} \leq \epsilon_{c}(x) \leq \bar{\epsilon}<\infty \quad \forall x \in \bar{\Omega}_{c} .
$$

The Lipschitz-continuity assumption (2.1) will be required in Theorem 3.8. The initial data $\boldsymbol{E}_{0}, \boldsymbol{H}_{0}$ and the desired electromagnetic fields $\boldsymbol{E}_{d}, \boldsymbol{H}_{d}$ are assumed to satisfy

$$
\boldsymbol{E}_{0}, \boldsymbol{E}_{d} \in \boldsymbol{H}_{0}(\mathrm{curl}) \quad \text { and } \quad \boldsymbol{H}_{0}, \boldsymbol{H}_{d} \in \boldsymbol{H}(\mathbf{c u r l}) .
$$

\section{MATHEMATICAL ANALYSIS}

We present a mathematical analysis for the optimal control problem $(\mathrm{P})$.

\subsection{Well-posedness}

Let us start by investigating the time-dependent Maxwell equations (1.1). The pivot space used in the theory is the following Hilbert space:

$$
\boldsymbol{X}:=\boldsymbol{L}^{2}(\Omega) \times \boldsymbol{L}^{2}(\Omega),
$$

equipped with the weighted scalar product

$$
((\boldsymbol{E}, \boldsymbol{H}),(\widehat{\boldsymbol{E}}, \widehat{\boldsymbol{H}}))_{\boldsymbol{X}}:=(\epsilon \boldsymbol{E}, \widehat{\boldsymbol{E}})_{\boldsymbol{L}^{2}(\Omega)}+(\mu \boldsymbol{H}, \widehat{\boldsymbol{H}})_{\boldsymbol{L}^{2}(\Omega)} \quad \forall(\boldsymbol{E}, \boldsymbol{H}),(\widehat{\boldsymbol{E}}, \widehat{\boldsymbol{H}}) \in \boldsymbol{X},
$$

which gives rise to the weighted norm

$$
\|(\boldsymbol{E}, \boldsymbol{H})\|_{\boldsymbol{X}}:=\sqrt{(\epsilon \boldsymbol{E}, \boldsymbol{E})_{\boldsymbol{L}^{2}(\Omega)}+(\mu \boldsymbol{H}, \boldsymbol{H})_{\boldsymbol{L}^{2}(\Omega)}} \quad \forall(\boldsymbol{E}, \boldsymbol{H}) \in \boldsymbol{X} .
$$

Since $\epsilon$ and $\mu$ are symmetric and positive definite, the bilinear form $(\cdot, \cdot)_{\boldsymbol{X}}: \boldsymbol{X} \times \boldsymbol{X} \rightarrow \mathbb{R}$ defines indeed a scalar product on $\boldsymbol{X}$. Let us define the Maxwell operator

$$
\mathcal{A}: D(\mathcal{A}) \rightarrow \boldsymbol{X}, \quad \mathcal{A}:=-\left(\begin{array}{ll}
\epsilon & 0 \\
0 & \mu
\end{array}\right)^{-1}\left(\begin{array}{cc}
0 & -\operatorname{curl} \\
\operatorname{curl} & 0
\end{array}\right)-\left(\begin{array}{cc}
\epsilon^{-1} \sigma & 0 \\
0 & 0
\end{array}\right),
$$

where the domain $D(\mathcal{A}) \subset \boldsymbol{X}$ is defined by

$$
D(\mathcal{A}):=\boldsymbol{H}_{0}(\operatorname{curl}) \times \boldsymbol{H}(\operatorname{curl}) .
$$

Employing the operator $\mathcal{A}$, the time-dependent Maxwell equations (1.1) can be equivalently formulated as the following Cauchy problem:

$$
\left\{\begin{aligned}
\left(\frac{\mathrm{d}}{\mathrm{d} t}-\mathcal{A}\right)(\boldsymbol{E}, \boldsymbol{H})(t) & =\left(\epsilon^{-1} \chi_{\Omega_{c}} \boldsymbol{u} a, 0\right) \quad \text { a.e. in }(0, T), \\
(\boldsymbol{E}, \boldsymbol{H})(0) & =\left(\boldsymbol{E}_{0}, \boldsymbol{H}_{0}\right) .
\end{aligned}\right.
$$


To justify the existence and uniqueness result for (3.3) ( $c f$. Leis [16]), let us first consider the following linear (unbounded) operator

$$
\mathcal{A}_{0}: \boldsymbol{X} \supset D\left(\mathcal{A}_{0}\right) \rightarrow \boldsymbol{X}, D\left(\mathcal{A}_{0}\right):=D(\mathcal{A}), \quad \mathcal{A}_{0}:=-\left(\begin{array}{cc}
\epsilon & 0 \\
0 & \mu
\end{array}\right)^{-1}\left(\begin{array}{cc}
0 & - \text { curl } \\
\text { curl } & 0
\end{array}\right) .
$$

This operator is densely defined and closed in X. Also, it satisfies

$$
\begin{aligned}
\left(\mathcal{A}_{0}(\boldsymbol{E}, \boldsymbol{H}),(\widehat{\boldsymbol{E}}, \widehat{\boldsymbol{H}})\right)_{\boldsymbol{X}} & =\left(\epsilon \epsilon^{-1} \operatorname{curl} \boldsymbol{H}, \widehat{\boldsymbol{E}}\right)_{\boldsymbol{L}^{2}(\Omega)}-\left(\mu \mu^{-1} \operatorname{curl} \boldsymbol{E}, \widehat{\boldsymbol{H}}\right)_{\boldsymbol{L}^{2}(\Omega)} \\
& =(\operatorname{curl} \boldsymbol{H}, \widehat{\boldsymbol{E}})_{\boldsymbol{L}^{2}(\Omega)}-(\operatorname{curl} \boldsymbol{E}, \widehat{\boldsymbol{H}})_{\boldsymbol{L}^{2}(\Omega)} \\
& =(\boldsymbol{H}, \operatorname{curl} \widehat{\boldsymbol{E}})_{\boldsymbol{L}^{2}(\Omega)}-(\boldsymbol{E}, \operatorname{curl} \widehat{\boldsymbol{H}})_{\boldsymbol{L}^{2}(\Omega)} \\
& =-\left(\epsilon \boldsymbol{E}, \epsilon^{-1} \operatorname{curl} \widehat{\boldsymbol{H}}\right)_{\boldsymbol{L}^{2}(\Omega)}+\left(\mu \boldsymbol{H}, \mu^{-1} \operatorname{curl} \widehat{\boldsymbol{E}}\right)_{\boldsymbol{L}^{2}(\Omega)}, \\
& =-\left((\boldsymbol{E}, \boldsymbol{H}), \mathcal{A}_{0}(\widehat{\boldsymbol{E}}, \widehat{\boldsymbol{H}})_{\boldsymbol{X}}, \quad \forall(\boldsymbol{E}, \boldsymbol{H}),(\widehat{\boldsymbol{E}}, \widehat{\boldsymbol{H}}) \in D\left(\mathcal{A}_{0}\right) .\right.
\end{aligned}
$$

where the third equality holds true since $\boldsymbol{E}, \widehat{\boldsymbol{E}} \in \boldsymbol{H}_{0}(\mathbf{c u r l})$ (see (3.27) and Rem. 3.28 in [19] for the corresponding integration by parts formula). Consequently, as $D\left(\mathcal{A}_{0}^{*}\right)=D\left(\mathcal{A}_{0}\right)$ (see Lem. A.2 in Appendix), the operator $\mathcal{A}_{0}$ is skew-adjoint, i.e., $\mathcal{A}_{0}^{*}=-\mathcal{A}_{0}$. Therefore, by Stone's theorem ([23], Thm. 10.8, p. 41), the operator $\mathcal{A}_{0}: D(\mathcal{A}) \rightarrow \boldsymbol{X}$ generates a $\mathcal{C}_{0}$-semigroup of unitary operators on $\boldsymbol{X}$. Now, the Maxwell operator $\mathcal{A}$ can be written as

$$
\mathcal{A}=\mathcal{A}_{0}+\mathcal{B}, \quad \text { with } \mathcal{B}: \boldsymbol{X} \rightarrow \boldsymbol{X}, \quad \mathcal{B}:=-\left(\begin{array}{cc}
\epsilon^{-1} \sigma & 0 \\
0 & 0
\end{array}\right)
$$

By Assumption 2.1, the operator $\mathcal{B}: \boldsymbol{X} \rightarrow \boldsymbol{X}$ is bounded and linear. Therefore, a well-known perturbation theorem ([23], Thm. 1.1, p. 76) implies that the Maxwell operator $\mathcal{A}: D(\mathcal{A}) \rightarrow \boldsymbol{X}$ is the infinitesimal generator of a $\mathcal{C}_{0}$-semigroup on $\boldsymbol{X}$, which we denote by $\left\{\mathbb{T}_{t}\right\}_{t \geq 0}$. For this reason, the Cauchy problem (3.3) admits a unique mild solution $(\boldsymbol{E}, \boldsymbol{H}) \in \mathcal{C}([0, T] ; \boldsymbol{X})$ defined by

$$
(\boldsymbol{E}, \boldsymbol{H})(t)=\mathbb{T}_{t}\left(\boldsymbol{E}_{0}, \boldsymbol{H}_{0}\right)+\int_{0}^{t} \mathbb{T}_{t-s}\left(\epsilon^{-1} \chi_{\Omega_{c}} \boldsymbol{u} a(s), 0\right) \mathrm{d} s \quad \forall t \in[0, T],
$$

satisfying

$$
\|(\boldsymbol{E}, \boldsymbol{H})\|_{\mathcal{C}([0, T] ; \boldsymbol{X})} \leq c\left(\left\|\left(\boldsymbol{E}_{0}, \boldsymbol{H}_{0}\right)\right\|_{\boldsymbol{X}}+\left\|\left(\epsilon^{-1} \chi_{\Omega_{c}} \boldsymbol{u} a, 0\right)\right\|_{L^{1}(0, T ; \boldsymbol{X})}\right),
$$

with a constant $c>0$ independent of $\boldsymbol{u}, a, \boldsymbol{E}_{0}$, and $\boldsymbol{H}_{0}$. We introduce the operator

$$
\mathcal{S}: \boldsymbol{L}^{2}\left(\Omega_{c}\right) \times L^{2}(0, T) \rightarrow \boldsymbol{X}, \quad(\boldsymbol{u}, a) \mapsto(\boldsymbol{E}, \boldsymbol{H})(T),
$$

that assigns to every $(\boldsymbol{u}, a) \in \boldsymbol{L}^{2}\left(\Omega_{c}\right) \times L^{2}(0, T)$ the unique mild solution $(\boldsymbol{E}, \boldsymbol{H})$ of (3.3) at the final time $t=T$. Employing this operator, we define the control-reduced objective functional $f: \boldsymbol{H}_{0}\left(\mathbf{c u r l} ; \Omega_{c}\right) \times L^{2}(0, T) \rightarrow \mathbb{R}$ by

$$
f(\boldsymbol{u}, a)=\frac{1}{2}\left\|\mathcal{S}(\boldsymbol{u}, a)-\left(\boldsymbol{E}_{d}, \boldsymbol{H}_{d}\right)\right\|_{\boldsymbol{X}}^{2}+\frac{\lambda_{\boldsymbol{u}}}{2}\|\operatorname{curl} \boldsymbol{u}\|_{\boldsymbol{L}^{2}\left(\Omega_{c}\right)}^{2}+\frac{\lambda_{a}}{2}\|a\|_{L^{2}(0, T)}^{2} .
$$

Note that the weighted norm $\|\cdot\|_{\boldsymbol{X}}$ is used in (3.8). Now, we reformulate the optimal control problem (P) into the following optimization problem in Hilbert spaces:

$$
\min _{(\boldsymbol{u}, a) \in \mathscr{U}^{\text {feas }} \times \mathscr{A}_{\text {feas }}} f(\boldsymbol{u}, a),
$$


where

$$
\begin{aligned}
\mathscr{U}^{\text {feas }} & :=\boldsymbol{H}_{0}\left(\operatorname{curl} ; \Omega_{c}\right) \cap \boldsymbol{H}\left(\operatorname{div}=0 ; \Omega_{c}\right) \\
\mathscr{A}^{\text {feas }} & :=\left\{a \in L^{2}(0, T) \mid a_{\min } \leq a(t) \leq a_{\max } \quad \text { a.e. in }(0, T)\right\} .
\end{aligned}
$$

We call a feasible control $\left(\boldsymbol{u}^{*}, a^{*}\right) \in \mathscr{U}^{\text {feas }} \times \mathscr{A}^{\text {feas }}$ optimal if and only if

$$
f\left(\boldsymbol{u}^{*}, a^{*}\right) \leq f(\boldsymbol{u}, a) \quad \forall(\boldsymbol{u}, a) \in \mathscr{U}^{\text {feas }} \times \mathscr{A}^{\text {feas }} .
$$

In the following, we exploit the compactness of the embedding

$$
\boldsymbol{H}_{0}\left(\operatorname{curl} ; \Omega_{c}\right) \cap \boldsymbol{H}\left(\operatorname{div} ; \Omega_{c}\right) \hookrightarrow \boldsymbol{L}^{2}\left(\Omega_{c}\right)
$$

(see [28]) and the Poincaré-Friedrichs-type inequality

$$
\begin{array}{r}
\|\boldsymbol{u}\|_{L^{2}\left(\Omega_{c}\right)} \leq c_{M}\left(\|\operatorname{curl} \boldsymbol{u}\|_{L^{2}\left(\Omega_{c}\right)}+\|\operatorname{div} \boldsymbol{u}\|_{L^{2}\left(\Omega_{c}\right)}\right) \\
\forall \boldsymbol{u} \in \boldsymbol{H}_{0}\left(\operatorname{curl} ; \Omega_{c}\right) \cap \boldsymbol{H}\left(\operatorname{div} ; \Omega_{c}\right)
\end{array}
$$

with a constant $c_{M}=c_{M}\left(\Omega_{c}\right)>0$. The Poincaré-Friedrichs-type inequality (3.10) follows from a standard contradiction argument ( $c f$. [19], Cor. 3.51) using the compact embedding (3.9) and

$$
\boldsymbol{D F}\left(\Omega_{c}\right):=\left\{\boldsymbol{u} \in \boldsymbol{H}_{0}\left(\operatorname{curl} ; \Omega_{c}\right) \cap \boldsymbol{H}\left(\operatorname{div} ; \Omega_{c}\right) \mid \operatorname{curl} \boldsymbol{u}=0, \operatorname{div} \boldsymbol{u}=0\right\}=\{0\} .
$$

Note that (3.11) holds true due to our assumption that $\Gamma_{c}$ is connected (see $\left.[2,24]\right)$. In this case, there exists no non-trivial Dirichlet harmonic field in $\Omega_{c}$.

Lemma 3.1. Let $\left\{\boldsymbol{u}_{k}\right\}_{k=0}^{\infty} \subset \mathscr{U}^{\text {feas }}$ and $\left\{a_{k}\right\}_{k=0}^{\infty} \subset L^{2}(0, T)$ such that

$$
\begin{array}{ll}
\boldsymbol{u}_{k} \rightarrow \boldsymbol{u}^{*} & \text { weakly in } \mathscr{U}^{\text {feas }}, \\
a_{k} \rightarrow a^{*} & \text { weakly in } L^{2}(0, T),
\end{array}
$$

for some $\left(\boldsymbol{u}^{*}, a^{*}\right) \in \mathscr{U}^{\text {feas }} \times \mathscr{A}^{\text {feas }}$. Then, it holds that

$$
\mathcal{S}\left(\boldsymbol{u}_{k}, a_{k}\right) \rightarrow \mathcal{S}\left(\boldsymbol{u}^{*}, a^{*}\right) \text { strongly in } \boldsymbol{X} .
$$

Proof. By definition, we have

$$
\begin{aligned}
\mathcal{S}\left(\boldsymbol{u}_{k}, a_{k}\right) & =\mathbb{T}_{T}\left(\boldsymbol{E}_{0}, \boldsymbol{H}_{0}\right)+\int_{0}^{T} \mathbb{T}_{T-s}\left(\epsilon^{-1} \chi_{\Omega_{c}} \boldsymbol{u}_{k} a_{k}(s), 0\right) \mathrm{d} s \\
& =\mathbb{T}_{T}\left(\boldsymbol{E}_{0}, \boldsymbol{H}_{0}\right)+\int_{0}^{T} a_{k}(s) \underbrace{\mathbb{T}_{T-s}\left(\epsilon^{-1} \chi_{\Omega_{c}} \boldsymbol{u}_{k}, 0\right)}_{=: g_{k}(s)} \mathrm{d} s .
\end{aligned}
$$

For the limit, we set

$$
g^{*}(s):=\mathbb{T}_{T-s}\left(\epsilon^{-1} \chi_{\Omega_{c}} \boldsymbol{u}^{*}, 0\right) \quad \forall s \in[0, T] .
$$

As $\left\{\mathbb{T}_{t}\right\}_{t \geq 0}$ is a $\mathcal{C}_{0}$-semigroup, there exist two constants $M \geq 1$ and $\omega \geq 0$ (see [23], Thm. 2.2, p. 4) such that

$$
\left\|\mathbb{T}_{t}\right\|_{\mathcal{L}(\boldsymbol{X})} \leq M \mathrm{e}^{\omega t} \quad \forall t \geq 0
$$


where $\mathcal{L}(\boldsymbol{X})$ denotes the space of bounded linear operators in $\boldsymbol{X}$. Consequently

$$
\begin{array}{r}
\max _{s \in[0, T]}\left\|g_{k}(s)-g^{*}(s)\right\|_{\boldsymbol{X}} \leq \max _{s \in[0, T]}\left\|\mathbb{T}_{T-s}\right\|_{\mathcal{L}(\boldsymbol{X})}\left\|\left(\epsilon^{-1} \chi_{\Omega_{c}}\left(\boldsymbol{u}_{k}-\boldsymbol{u}^{*}\right), 0\right)\right\|_{\boldsymbol{X}} \\
\underbrace{\leq}_{(2.2)} \underline{\epsilon}^{-1 / 2} \max _{s \in[0, T]}\left\|\mathbb{T}_{T-s}\right\|_{\mathcal{L}(\boldsymbol{X})}\left\|\boldsymbol{u}_{k}-\boldsymbol{u}^{*}\right\|_{\boldsymbol{L}^{2}\left(\Omega_{c}\right)} \\
\leq \underline{\epsilon}^{-1 / 2} M \mathrm{e}^{\omega T}\left\|\boldsymbol{u}_{k}-\boldsymbol{u}^{*}\right\|_{\boldsymbol{L}^{2}\left(\Omega_{c}\right)} .
\end{array}
$$

Now, since the embedding (3.9) is compact, the weak convergence (3.12) implies that

$$
\boldsymbol{u}_{k} \rightarrow \boldsymbol{u}^{*} \quad \text { in } \boldsymbol{L}^{2}\left(\Omega_{c}\right) \underset{(3.15)}{\Longrightarrow} g_{k} \rightarrow g^{*} \quad \text { in } \mathcal{C}([0, T] ; \boldsymbol{X})
$$

The above strong convergence together with the weak convergence (3.13) yields that

$$
\int_{0}^{T} a_{k}(s) g_{k}(s) \mathrm{d} s \rightarrow \int_{0}^{T} a^{*}(s) g^{*}(s) \mathrm{d} s \quad \text { in } \boldsymbol{X},
$$

from which (3.14) follows.

As a consequence of Lemma 3.1 and the Poincaré-Friedrichs-type inequality (3.10), we obtain the following existence result:

Theorem 3.2. The optimal control problem (P) admits an optimal solution.

\subsection{KKT Theory}

This section is devoted to the KKT theory for $(\mathrm{P})$. Let us first derive an explicit formula for the first-oder derivative of the objective functional.

Lemma 3.3. Let $(\boldsymbol{u}, a) \in \boldsymbol{H}_{0}\left(\boldsymbol{c u r l} ; \Omega_{c}\right) \times L^{2}(0, T)$ with the associated mild solution $(\boldsymbol{E}, \boldsymbol{H}) \in \mathcal{C}([0, T], \boldsymbol{X})$ of (3.3). Let $(\boldsymbol{K}, \boldsymbol{Q}) \in \mathcal{C}([0, T], \boldsymbol{X})$ be defined as follows:

$$
(\boldsymbol{K}, \boldsymbol{Q})(t):=\mathbb{T}_{T-t}^{*}\left(\boldsymbol{E}(T)-\boldsymbol{E}_{d}, \boldsymbol{H}(T)-\boldsymbol{H}_{d}\right) \quad \forall t \in[0, T],
$$

where $\mathbb{T}_{\tau}^{*}: \boldsymbol{X} \rightarrow \boldsymbol{X}$, for every $\tau \geq 0$, denote the adjoint operator of $\mathbb{T}_{\tau}$. Then, it holds that

$$
\begin{array}{r}
f^{\prime}(\boldsymbol{u}, a)(\delta \boldsymbol{u}, \delta a)=\int_{0}^{T} \int_{\Omega_{c}} \boldsymbol{K}(x, t) \cdot(\boldsymbol{u}(x) \delta a(t)+\delta \boldsymbol{u}(x) a(t)) \mathrm{d} x \mathrm{~d} t \\
\quad+\lambda_{\boldsymbol{u}} \int_{\Omega_{c}} \operatorname{curl} \boldsymbol{u} \cdot \operatorname{curl} \delta \boldsymbol{u} \mathrm{d} x+\lambda_{a} \int_{0}^{T} a \delta a \mathrm{~d} t
\end{array}
$$

for all $(\delta \boldsymbol{u}, \delta a) \in \boldsymbol{H}_{0}\left(\operatorname{curl} ; \Omega_{c}\right) \times L^{2}(0, T)$.

Proof. Let $(\delta \boldsymbol{u}, \delta a) \in \boldsymbol{H}_{0}\left(\mathbf{c u r l} ; \Omega_{c}\right) \times L^{2}(0, T)$. We only carry out the calculations for the first term of the objective functional $f$, which is denoted by

$$
\tilde{f}(\boldsymbol{u}, a):=\frac{1}{2}\left\|\mathcal{S}(\boldsymbol{u}, a)-\left(\boldsymbol{E}_{d}, \boldsymbol{H}_{d}\right)\right\|_{\boldsymbol{X}}^{2} .
$$


Using the definition of the mild solution (3.5) and the chain rule for the derivative of $\tilde{f}$, we deduce that

$$
\begin{aligned}
& \tilde{f}^{\prime}(\boldsymbol{u}, a)(\delta \boldsymbol{u}, \delta a)=\left(\mathcal{S}(\boldsymbol{u}, a)-\left(\boldsymbol{E}_{d}, \boldsymbol{H}_{d}\right), \mathcal{S}^{\prime}(\boldsymbol{u}, a)(\delta \boldsymbol{u}, \delta a)\right)_{\boldsymbol{X}} \\
& =\left(\left(\boldsymbol{E}(T)-\boldsymbol{E}_{d}, \boldsymbol{H}(T)-\boldsymbol{H}_{d}\right), \mathcal{S}^{\prime}(\boldsymbol{u}, a)(\delta \boldsymbol{u}, \delta a)\right)_{\boldsymbol{X}} \\
& =\left(\left(\boldsymbol{E}(T)-\boldsymbol{E}_{d}, \boldsymbol{H}(T)-\boldsymbol{H}_{d}\right), \int_{0}^{T} \mathbb{T}_{T-t}\left(\epsilon^{-1} \chi_{\Omega_{c}}(\boldsymbol{u} \delta a(t)+\delta \boldsymbol{u} a(t)), 0\right) \mathrm{d} t\right)_{\boldsymbol{X}} \\
& =\int_{0}^{T}\left(\left(\boldsymbol{E}(T)-\boldsymbol{E}_{d}, \boldsymbol{H}(T)-\boldsymbol{H}_{d}\right), \mathbb{T}_{T-t}\left(\epsilon^{-1} \chi_{\Omega_{c}}(\boldsymbol{u} \delta a(t)+\delta \boldsymbol{u} a(t)), 0\right)\right)_{\boldsymbol{X}} \mathrm{d} t \\
& =\int_{0}^{T}\left(\mathbb{T}_{T-t}^{*}\left(\boldsymbol{E}(T)-\boldsymbol{E}_{d}, \boldsymbol{H}(T)-\boldsymbol{H}_{d}\right),\left(\epsilon^{-1} \chi_{\Omega_{c}}(\boldsymbol{u} \delta a(t)+\delta \boldsymbol{u} a(t)), 0\right)\right)_{\boldsymbol{X}} \mathrm{d} t \\
& \underbrace{=}_{(3.16)} \int_{0}^{T}\left((\boldsymbol{K}, \boldsymbol{Q}),\left(\epsilon^{-1} \chi_{\Omega_{c}}(\boldsymbol{u} \delta a(t)+\delta \boldsymbol{u} a(t)), 0\right)\right)_{\boldsymbol{X}} \mathrm{d} t \\
& \underbrace{=}_{(3.1)} \int_{0}^{T} \int_{\Omega_{c}} \boldsymbol{K}(x, t) \cdot(\boldsymbol{u}(x) \delta a(t)+\delta \boldsymbol{u}(x) a(t)) \mathrm{d} x \mathrm{~d} t .
\end{aligned}
$$

The above equality yields immediately the formula (3.17).

Definition 3.4 (adjoint state). Let $\left(\boldsymbol{u}^{*}, a^{*}\right) \in \mathscr{U}^{\text {feas }} \times \mathscr{A}^{\text {feas }}$ be an optimal control of (P) with the associated mild solution $(\boldsymbol{E}, \boldsymbol{H}) \in \mathcal{C}([0, T], \boldsymbol{X})$ of $(3.3)$. Then, we call $\left(\boldsymbol{K}^{*}, \boldsymbol{Q}^{*}\right) \in \mathcal{C}([0, T] ; \boldsymbol{X})$, defined by

$$
\left(\boldsymbol{K}^{*}, \boldsymbol{Q}^{*}\right)(t):=\mathbb{T}_{T-t}^{*}\left(\boldsymbol{E}^{*}(T)-\boldsymbol{E}_{d}, \boldsymbol{H}^{*}(T)-\boldsymbol{H}_{d}\right) \quad \forall t \in[0, T],
$$

the adjoint state associated with the optimal control $\left(\boldsymbol{u}^{*}, a^{*}\right)$.

Remark 3.5. The third equality in the proof of Lemma 3.3 holds under the use of the weighted scalar product $(\cdot, \cdot)_{\boldsymbol{X}}$. If the weight functions $\epsilon$ and $\mu$ were neglected in the objective functional, i.e., if

$$
f(\boldsymbol{u}, a)=\frac{1}{2}\left\|\mathcal{S}(\boldsymbol{u}, a)-\left(\boldsymbol{E}_{d}, \boldsymbol{H}_{d}\right)\right\|_{\boldsymbol{L}^{2}(\Omega) \times \boldsymbol{L}^{2}(\Omega)}^{2}+\frac{\lambda_{u}}{2}\|\operatorname{curl} \boldsymbol{u}\|_{\boldsymbol{L}^{2}\left(\Omega_{c}\right)}^{2}+\frac{\lambda_{a}}{2}\|a\|_{L^{2}(0, T)}^{2},
$$

then we would have to define the adjoint state as follows:

$$
\left(\boldsymbol{K}^{*}, \boldsymbol{Q}^{*}\right)(t)=\mathbb{T}_{T-t}^{*}\left(\epsilon^{-1}\left(\boldsymbol{E}^{*}(T)-\boldsymbol{E}_{d}\right), \mu^{-1}\left(\boldsymbol{H}^{*}(T)-\boldsymbol{H}_{d}\right)\right) \quad \forall t \in[0, T],
$$

as the weighted scalar product $(\cdot, \cdot)_{\boldsymbol{X}}$ is used here. In this case, we could not extract the strong regularity for the adjoint state (see Thm. 3.8). This is the main reason for including the weight functions $\epsilon$ and $\mu$ in the objective functional.

For the later use, let us define the projection

$$
\mathbb{P}_{\left[a_{\min }, a_{\max }\right]}: \mathbb{R} \rightarrow\left[a_{\min }, a_{\max }\right], \quad \mathbb{P}_{\left[a_{\min }, a_{\max }\right]}\{a\}=\min \left\{a_{\max }, \max \left\{a, a_{\min }\right\}\right\} .
$$

In the upcoming theorem, we prove an important characterization for the optimal control of $(\mathrm{P})$, where the main tool for the proof is the following Helmholtz-type decomposition:

Lemma 3.6. The Hilbert spaces $\boldsymbol{L}^{2}\left(\Omega_{c}\right)$ and $\boldsymbol{H}_{0}\left(\mathbf{c u r l} ; \Omega_{c}\right)$ admit the following decompositions:

$$
\begin{aligned}
\boldsymbol{L}^{2}\left(\Omega_{c}\right) & =\nabla H_{0}^{1}\left(\Omega_{c}\right) \oplus \boldsymbol{H}\left(\operatorname{div}=0 ; \Omega_{c}\right), \\
\boldsymbol{H}_{0}\left(\operatorname{curl} ; \Omega_{c}\right) & =\nabla H_{0}^{1}\left(\Omega_{c}\right) \oplus \boldsymbol{H}_{0}\left(\operatorname{curl} ; \Omega_{c}\right) \cap \boldsymbol{H}\left(\operatorname{div}=0 ; \Omega_{c}\right) .
\end{aligned}
$$


Note that (3.18) and (3.19) follow from the classical Hilbert projection theorem since $\nabla H_{0}^{1}\left(\Omega_{c}\right) \subset \boldsymbol{L}^{2}\left(\Omega_{c}\right)$ and $\nabla H_{0}^{1}\left(\Omega_{c}\right) \subset \boldsymbol{H}_{0}\left(\operatorname{curl} ; \Omega_{c}\right)$ are closed.

Theorem 3.7. Let $\left(\boldsymbol{u}^{*}, a^{*}\right) \in \mathscr{U}^{\text {feas }} \times \mathscr{A}^{\text {feas }}$ be an optimal control of $(\mathrm{P})$ with the associated adjoint state $\left(\boldsymbol{K}^{*}, \boldsymbol{Q}^{*}\right) \in \mathcal{C}([0, T], \boldsymbol{X})$. Then, there exists a unique function $\varphi \in H_{0}^{1}\left(\Omega_{c}\right)$ such that

$$
\left\{\begin{aligned}
\left(\operatorname{curl} \boldsymbol{u}^{*}, \operatorname{curl} \boldsymbol{v}\right)_{\boldsymbol{L}^{2}\left(\Omega_{c}\right)}+(\boldsymbol{v}, \nabla \varphi)_{\boldsymbol{L}^{2}\left(\Omega_{c}\right)} & =-\lambda_{\boldsymbol{u}}^{-1}\left(\boldsymbol{z}_{a}^{*}, \boldsymbol{v}\right)_{\boldsymbol{L}^{2}\left(\Omega_{c}\right)}, \\
\left(\boldsymbol{u}^{*}, \nabla \phi\right)_{\boldsymbol{L}^{2}\left(\Omega_{c}\right)} & =0
\end{aligned}\right.
$$

for all $(\boldsymbol{v}, \phi) \in \boldsymbol{H}_{0}\left(\operatorname{curl} ; \Omega_{c}\right) \times H_{0}^{1}\left(\Omega_{c}\right)$, and

$$
a^{*}(t)=\mathbb{P}_{\left[a_{\min }, a_{\max }\right]}\left\{-\frac{z_{\boldsymbol{u}}^{*}(t)}{\lambda_{a}}\right\} \quad \text { a.e. in }(0, T),
$$

where $\boldsymbol{z}_{a}^{*}:=\left(\boldsymbol{K}^{*}, a^{*}\right)_{L^{2}(0, T)}$ and $z_{\boldsymbol{u}}^{*}:=\left(\boldsymbol{K}^{*}, \boldsymbol{u}^{*}\right)_{\boldsymbol{L}^{2}\left(\Omega_{c}\right)}$.

Proof. As the feasible control set $\mathscr{U}^{\text {feas }} \times \mathscr{A}^{\text {feas }} \subset \boldsymbol{H}_{0}\left(\mathbf{c u r l} ; \Omega_{c}\right) \times L^{2}(0, T)$ is convex and the objective functional $f: \boldsymbol{H}_{0}\left(\mathbf{c u r l} ; \Omega_{c}\right) \times L^{2}(0, T) \rightarrow \mathbb{R}$ is Gâteaux differentiable, the optimal control $\left(\boldsymbol{u}^{*}, a^{*}\right)$ satisfies the following variational inequality:

$$
f^{\prime}\left(\boldsymbol{u}^{*}, a^{*}\right)\left(\boldsymbol{u}-\boldsymbol{u}^{*}, a-a^{*}\right) \geq 0 \quad \forall(\boldsymbol{u}, a) \in \mathscr{U}^{\text {feas }} \times \mathscr{A}^{\text {feas }} .
$$

We refer to ([25], Lem. 2.21) for the variational inequality (3.22). Setting $a=a^{*}$ in (3.22) and using the fact that $\mathscr{U}^{\text {feas }}$ is a linear space, it follows immediately that

$$
f^{\prime}\left(\boldsymbol{u}^{*}, a^{*}\right)(\boldsymbol{u}, 0)=0 \quad \forall \boldsymbol{u} \in \mathscr{U}^{\text {feas }} .
$$

Then, using the formula (3.17), the above variational equality is equivalent to

$$
\int_{\Omega_{c}} \boldsymbol{z}_{a}^{*} \cdot \boldsymbol{u} \mathrm{d} x+\lambda_{\boldsymbol{u}} \int_{\Omega_{c}} \operatorname{curl} \boldsymbol{u}^{*} \cdot \operatorname{curl} \boldsymbol{u} \mathrm{d} x=0 \quad \forall \boldsymbol{u} \in \mathscr{U}^{\text {feas }} .
$$

In view of the first Helmholtz-type decomposition (3.18), we find a unique function $\varphi \in H_{0}^{1}\left(\Omega_{c}\right)$ such that

$$
\lambda_{\boldsymbol{u}}^{-1} \boldsymbol{z}_{a}^{*}+\nabla \varphi \in \boldsymbol{H}\left(\operatorname{div}=0 ; \Omega_{c}\right),
$$

or equivalently

$$
\left(\lambda_{\boldsymbol{u}}^{-1} \boldsymbol{z}_{a}^{*}+\nabla \varphi, \nabla \phi\right)_{\boldsymbol{L}^{2}\left(\Omega_{c}\right)}=0 \quad \forall \phi \in H_{0}^{1}\left(\Omega_{c}\right) .
$$

As $(\boldsymbol{u}, \nabla \varphi)_{\boldsymbol{L}^{2}\left(\Omega_{c}\right)}=0$ holds for all $\boldsymbol{u} \in \mathscr{U}^{\text {feas }}$, we see that (3.23) is equivalent to

$$
\left(\operatorname{curl} \boldsymbol{u}^{*}, \operatorname{curl} \boldsymbol{u}\right)_{\boldsymbol{L}^{2}\left(\Omega_{c}\right)}=-\left(\lambda_{\boldsymbol{u}}^{-1} \boldsymbol{z}_{a}^{*}+\nabla \varphi, \boldsymbol{u}\right)_{\boldsymbol{L}^{2}\left(\Omega_{c}\right)} \quad \forall \boldsymbol{u} \in \mathscr{U}^{\text {feas }} .
$$

Using (3.24) and since $\operatorname{curl} \nabla \equiv 0$, it follows from (3.25) that

$$
\begin{array}{r}
\left(\operatorname{curl} \boldsymbol{u}^{*}, \operatorname{curl}(\nabla \phi+\boldsymbol{u})\right)_{\boldsymbol{L}^{2}\left(\Omega_{c}\right)}=-\left(\lambda_{\boldsymbol{u}}^{-1} \boldsymbol{z}_{a}^{*}+\nabla \varphi, \nabla \phi+\boldsymbol{u}\right)_{\boldsymbol{L}^{2}\left(\Omega_{c}\right)} \\
\forall \phi \in H_{0}^{1}\left(\Omega_{c}\right), \forall \boldsymbol{u} \in \mathscr{U}^{\text {feas }} .
\end{array}
$$

Now, in view of the second Helmholtz-type decomposition (3.19), (3.26) implies

$$
\left(\operatorname{curl} \boldsymbol{u}^{*}, \operatorname{curl} \boldsymbol{v}\right)_{\boldsymbol{L}^{2}\left(\Omega_{c}\right)}=-\left(\lambda_{\boldsymbol{u}}^{-1} \boldsymbol{z}_{a}^{*}+\nabla \varphi, \boldsymbol{v}\right)_{\boldsymbol{L}^{2}\left(\Omega_{c}\right)} \quad \forall \boldsymbol{v} \in \boldsymbol{H}_{0}\left(\operatorname{curl} ; \Omega_{c}\right),
$$


or equivalently

$$
\left(\operatorname{curl} \boldsymbol{u}^{*}, \operatorname{curl} \boldsymbol{v}\right)_{\boldsymbol{L}^{2}\left(\Omega_{c}\right)}+(\nabla \varphi, \boldsymbol{v})_{\boldsymbol{L}^{2}\left(\Omega_{c}\right)}=-\lambda_{\boldsymbol{u}}^{-1}\left(\boldsymbol{z}_{a}^{*}, \boldsymbol{v}\right)_{\boldsymbol{L}^{2}\left(\Omega_{c}\right)} \quad \forall \boldsymbol{v} \in \boldsymbol{H}_{0}\left(\operatorname{curl} ; \Omega_{c}\right) .
$$

In conclusion, $\left(\boldsymbol{u}^{*}, \varphi\right)$ solves the magnetostatic saddle point problem (3.20).

On the other hand, setting $\boldsymbol{u}=\boldsymbol{u}^{*}$ in the variational inequality (3.22) yields that

$$
f^{\prime}\left(\boldsymbol{u}^{*}, a^{*}\right)\left(0, a-a^{*}\right) \geq 0 \quad \forall a \in \mathscr{A}^{\text {feas }} .
$$

Then, using again the formula (3.17), we see that (3.27) is equivalent to

$$
\int_{0}^{T}\left(\frac{1}{\lambda_{a}} z_{\boldsymbol{u}}^{*}(t)+a^{*}(t)\right)\left(a(t)-a^{*}(t)\right) \mathrm{d} t \geq 0 \quad \forall a \in \mathscr{A}^{\text {feas }} .
$$

By standard arguments, a pointwise evaluation of this variational inequality yields the projection formula (3.21).

Now, with Theorem 3.7 at hand, we are able to prove the following higher regularity result:

Theorem 3.8. Let $\left(\boldsymbol{u}^{*}, a^{*}\right) \in \mathscr{U}^{\text {feas }} \times \mathscr{A}^{\text {feas }}$ be an optimal control of $(\mathrm{P})$ with the associated mild solution $\left(\boldsymbol{E}^{*}, \boldsymbol{H}^{*}\right) \in \mathcal{C}([0, T], \boldsymbol{X})$ of $(3.3)$ and the adjoint state $\left(\boldsymbol{K}^{*}, \boldsymbol{Q}^{*}\right) \in \mathcal{C}([0, T], \boldsymbol{X})$. Then, it holds that

$$
\begin{array}{r}
\boldsymbol{u}^{*}, \operatorname{curl} \boldsymbol{u}^{*} \in \boldsymbol{H}^{\frac{1}{2}}\left(\Omega_{c}\right), \\
a^{*} \in \mathcal{C}^{0,1}[0, T], \\
\left(\boldsymbol{E}^{*}, \boldsymbol{H}^{*}\right),\left(\boldsymbol{K}^{*}, \boldsymbol{Q}^{*}\right) \in \mathcal{C}([0, T] ; D(\mathcal{A})) \cap \mathcal{C}^{1}([0, T] ; \boldsymbol{X}) .
\end{array}
$$

In particular, $\left(\boldsymbol{E}^{*}, \boldsymbol{H}^{*}\right) \in \mathcal{C}([0, T] ; D(\mathcal{A})) \cap \mathcal{C}^{1}([0, T] ; \boldsymbol{X})$ is the (classical) solution of the Maxwell equations (1.1), i.e., it satisfies (1.1) for a.e. $x \in \Omega$ and all $t \in[0, T]$.

Proof. As $\boldsymbol{u}^{*} \in \mathscr{U}^{\text {feas }}=\boldsymbol{H}_{0}\left(\mathbf{c u r l} ; \Omega_{c}\right) \cap \boldsymbol{H}\left(\operatorname{div}=0 ; \Omega_{c}\right)$ satisfies the system (3.20), it follows from the distributional definition of the curl-operator and the vanishing tangential boundary condition that $\operatorname{curl} \boldsymbol{u}^{*} \in$ $\boldsymbol{H}\left(\operatorname{curl}, \Omega_{c}\right) \cap \boldsymbol{H}_{0}\left(\operatorname{div}=0, \Omega_{c}\right)$. Thus, employing the embedding results

$$
\begin{aligned}
& \boldsymbol{H}_{0}\left(\operatorname{curl} ; \Omega_{c}\right) \cap \boldsymbol{H}\left(\operatorname{div} ; \Omega_{c}\right) \hookrightarrow \boldsymbol{H}^{\frac{1}{2}}\left(\Omega_{c}\right) \\
& \boldsymbol{H}\left(\operatorname{curl} ; \Omega_{c}\right) \cap \boldsymbol{H}_{0}\left(\operatorname{div} ; \Omega_{c}\right) \hookrightarrow \boldsymbol{H}^{\frac{1}{2}}\left(\Omega_{c}\right)
\end{aligned}
$$

(see [5], Thm. 2), we obtain (3.28a).

Now, in view of the regularity $\boldsymbol{K}^{*} \in \mathcal{C}\left([0, T] ; \boldsymbol{L}^{2}(\Omega)\right)$, the function $z_{\boldsymbol{u}}^{*}:[0 ; T] \rightarrow \mathbb{R}, z_{\boldsymbol{u}}^{*}(t)=\left(\boldsymbol{K}^{*}(t), \boldsymbol{u}^{*}\right)_{\boldsymbol{L}^{2}\left(\Omega_{c}\right)}$ is continuous. Thus, the projection formula (3.21) implies

$$
a^{*} \in \mathcal{C}[0, T]
$$

Furthermore, by the assumptions (2.1) and (2.2), we have that $\epsilon_{\mid \Omega_{c}}^{-1}=\epsilon_{c}^{-1} I_{d}$, with $\epsilon_{c}^{-1} \in \mathcal{C}^{0,1}\left(\bar{\Omega}_{c}\right)$ and consequently, since $\boldsymbol{u}^{*} \in \boldsymbol{H}_{0}\left(\operatorname{curl} ; \Omega_{c}\right)$, it follows that

$$
\epsilon_{c}^{-1} \boldsymbol{u}^{*} \in \boldsymbol{H}_{0}\left(\operatorname{curl} ; \Omega_{c}\right)
$$

The proof for this product regularity property is given in the appendix (Lem. A.1). Now, as $\epsilon_{c}^{-1} \boldsymbol{u}^{*}$ has a vanishing tangential trace on the boundary $\Gamma_{c}$, its zero extension into $\Omega$ is then well-defined as an element of $\boldsymbol{H}_{0}(\mathbf{c u r l})$ (cf. [19], Lem. 5.3), and so

$$
\epsilon^{-1} \chi_{\Omega_{c}} \boldsymbol{u}^{*} \in \boldsymbol{H}_{0}(\operatorname{curl})
$$


Summarizing from (3.29) and (3.30), we have

$$
\left(\epsilon^{-1} \chi_{\Omega_{c}} \boldsymbol{u}^{*} a^{*}, 0\right) \in \mathcal{C}([0, T] ; D(\mathcal{A})) .
$$

Due to the above regularity and $\left(\boldsymbol{E}_{0}, \boldsymbol{H}_{0}\right) \in D(\mathcal{A})$ (see (2.3)), we may use the well-known result from the semigroup theory ([23], Cor. 2.6, p. 108) to conclude that the mild solution

$$
\left(\boldsymbol{E}^{*}, \boldsymbol{H}^{*}\right)(t)=\mathbb{T}_{t}\left(\boldsymbol{E}_{0}, \boldsymbol{H}_{0}\right)+\int_{0}^{t} \mathbb{T}_{t-s}\left(\epsilon^{-1} \chi_{\Omega_{c}} \boldsymbol{u}^{*} a^{*}(s), 0\right) \mathrm{d} s
$$

is a classical solution:

$$
\left(\boldsymbol{E}^{*}, \boldsymbol{H}^{*}\right) \in \mathcal{C}([0, T] ; D(\mathcal{A})) \cap \mathcal{C}^{1}([0, T] ; \boldsymbol{X}) .
$$

The regularity (3.31) together with the assumption $\left(\boldsymbol{E}_{d}, \boldsymbol{H}_{d}\right) \in D(\mathcal{A})$ (see (2.3)) yields that

$$
\left(\boldsymbol{E}^{*}(T)-\boldsymbol{E}_{d}, \boldsymbol{H}^{*}(T)-\boldsymbol{H}_{d}\right) \in D(\mathcal{A})=D\left(\mathcal{A}^{*}\right) .
$$

Now, as the adjoint operator $\mathcal{A}^{*}$ is the infinitesimal generator of $\left\{\mathbb{T}_{t}^{*}\right\}_{t \geq 0}$ (see [23], Cor. 10.6, p. 41), the regularity property (3.32) implies that the adjoint state, given by

$$
\left(\boldsymbol{K}^{*}, \boldsymbol{Q}^{*}\right)(t)=\mathbb{T}_{T-t}^{*}\left(\boldsymbol{E}^{*}(T)-\boldsymbol{E}_{d}, \boldsymbol{H}^{*}(T)-\boldsymbol{H}_{d}\right), \quad \forall t \in[0, T]
$$

satisfies

$$
\left(\boldsymbol{K}^{*}, \boldsymbol{Q}^{*}\right) \in \mathcal{C}([0, T] ; D(\mathcal{A})) \cap \mathcal{C}^{1}([0, T] ; \boldsymbol{X}) .
$$

In view of (3.33), it follows that $z_{\boldsymbol{u}}^{*}=\left(\boldsymbol{K}^{*}, \boldsymbol{u}^{*}\right)_{\boldsymbol{L}^{2}\left(\Omega_{c}\right)} \in \mathcal{C}^{1}[0, T]$. Thus, the projection formula (3.21) implies finally the Lipschitz continuity: $a^{*} \in \mathcal{C}^{0,1}[0, T]$. This completes the proof.

Note that, if the control domain $\Omega_{c}$ is Lipschitz-polyhedral, then $\boldsymbol{u}^{*}$ enjoys the following higher regularity properties:

$$
\boldsymbol{u}^{*}, \operatorname{curl} \boldsymbol{u}^{*} \in \boldsymbol{H}^{\frac{1}{2}+\delta_{c}}\left(\Omega_{c}\right),
$$

for some $\delta_{c} \in(0,0.5]$. If $\Omega_{c}$ is convex or of class $\mathcal{C}^{1,1}$, then the regularity property holds true for $\delta_{c}=0.5$. This follows from the embedding results ([2], Prop. 3.7 and Thm. 2.17).

A main consequence of Theorem 3.7 and 3.8 is the following optimality system for $(\mathrm{P})$ :

Theorem 3.9. Let $\left(\boldsymbol{u}^{*}, a^{*}\right) \in \mathscr{U}^{\text {feas }} \times \mathscr{A}^{\text {feas }}$ be an optimal control of $(\mathrm{P})$ with the associated optimal electromagnetic fields $\left(\boldsymbol{E}^{*}, \boldsymbol{H}^{*}\right) \in \mathcal{C}([0, T] ; D(\mathcal{A})) \cap \mathcal{C}^{1}([0, T] ; \boldsymbol{X})$. Then, there exists a unique triple $\left(\varphi, \boldsymbol{K}^{*}, \boldsymbol{Q}^{*}\right) \in$ $H_{0}^{1}\left(\Omega_{c}\right) \times \mathcal{C}([0, T] ; D(\mathcal{A})) \cap \mathcal{C}^{1}([0, T] ; \boldsymbol{X})$ such that

$$
\begin{aligned}
& \begin{cases}\epsilon \boldsymbol{E}_{t}^{*}-\operatorname{curl} \boldsymbol{H}^{*}+\sigma \boldsymbol{E}^{*}=\chi_{\Omega_{c}} \boldsymbol{u}^{*} a^{*} & \text { in } \Omega \times(0, T), \\
\mu \boldsymbol{H}_{t}^{*}+\operatorname{curl} \boldsymbol{E}^{*}=0 & \text { in } \Omega \times(0, T), \\
\boldsymbol{E}^{*} \times \boldsymbol{n}=0 & \text { in } \Gamma \times(0, T), \\
\boldsymbol{E}^{*}(0)=\boldsymbol{E}_{0} & \text { in } \Omega, \\
\boldsymbol{H}^{*}(0)=\boldsymbol{H}_{0} & \text { in } \Omega,\end{cases} \\
& \begin{cases}\epsilon \boldsymbol{K}_{t}^{*}-\operatorname{curl} \boldsymbol{Q}^{*}-\sigma \boldsymbol{K}^{*}=0 & \text { in } \Omega \times(0, T), \\
\mu \boldsymbol{Q}_{t}^{*}+\operatorname{curl} \boldsymbol{K}^{*}=0 & \text { in } \Omega \times(0, T), \\
\boldsymbol{K}^{*} \times \boldsymbol{n}=0 & \text { in } \Gamma \times(0, T), \\
\boldsymbol{K}^{*}(T)=\boldsymbol{E}^{*}(T)-\boldsymbol{E}_{d} & \text { in } \Omega, \\
\boldsymbol{Q}^{*}(T)=\boldsymbol{H}^{*}(T)-\boldsymbol{H}_{d} & \text { in } \Omega,\end{cases} \\
& \left\{\begin{aligned}
\operatorname{curl} \operatorname{curl} \boldsymbol{u}^{*}+\nabla \varphi & =-\lambda_{\boldsymbol{u}}^{-1}\left(\boldsymbol{K}^{*}, a^{*}\right)_{L^{2}(0, T)} & & \text { in } \Omega_{c}, \\
\operatorname{div} \boldsymbol{u}^{*} & =0 & & \text { in } \Omega_{c}, \\
\boldsymbol{u}^{*} \times \boldsymbol{n}_{c} & =0 & & \text { on } \Gamma_{c},
\end{aligned}\right. \\
& a^{*}(t)=\mathbb{P}_{\left[a_{\min }, a_{\max }\right]}\left(-\frac{1}{\lambda_{a}} \int_{\Omega_{c}} \boldsymbol{K}^{*}(x, t) \cdot \boldsymbol{u}^{*}(x) \mathrm{d} x\right) \quad \forall t \in[0, T] .
\end{aligned}
$$


Note that the forward-backward Maxwell system (3.34a)-(3.34b) holds in the strong sense, whereas (3.34c) is satisfied by the optimal current density $\boldsymbol{u}^{*}$ and the Lagrange multiplier $\varphi$ only in the weak sense; see its mixed variational formulation in (3.20).

Proof. It remains to prove that the adjoint state $\left(\boldsymbol{K}^{*}, \boldsymbol{Q}^{*}\right) \in \mathcal{C}([0, T] ; D(\mathcal{A})) \cap \mathcal{C}^{1}([0, T] ; \boldsymbol{X})$ is the solution of $(3.34 \mathrm{~b})$. Indeed, we have that

$$
\begin{aligned}
\frac{\mathrm{d}}{\mathrm{d} t}\left(\boldsymbol{K}^{*}, \boldsymbol{Q}^{*}\right)(t) & =\frac{\mathrm{d}}{\mathrm{d} t} \mathbb{T}_{T-t}^{*}\left(\boldsymbol{E}^{*}(T)-\boldsymbol{E}_{d}, \boldsymbol{H}^{*}(T)-\boldsymbol{H}_{d}\right) \\
& =-\mathcal{A}^{*} \mathbb{T}_{T-t}^{*}\left(\boldsymbol{E}^{*}(T)-\boldsymbol{E}_{d}, \boldsymbol{H}^{*}(T)-\boldsymbol{H}_{d}\right) \\
& =-\mathcal{A}^{*}\left(\boldsymbol{K}^{*}, \boldsymbol{Q}^{*}\right)(t) \quad \forall t \in(0, T),
\end{aligned}
$$

since the adjoint operator $\mathcal{A}^{*}$ is the infinitesimal generator of $\left\{\mathbb{T}_{t}^{*}\right\}_{t \geq 0}$ and $\left(\boldsymbol{E}^{*}(T)-\boldsymbol{E}_{d}, \boldsymbol{H}^{*}(T)-\boldsymbol{H}_{d}\right) \in D(\mathcal{A})=$ $D\left(\mathcal{A}^{*}\right)$. Furthermore, $\left(\boldsymbol{K}^{*}, \boldsymbol{Q}^{*}\right)(T)=\mathbb{T}_{0}^{*}\left(\boldsymbol{E}^{*}(T)-\boldsymbol{E}_{d}, \boldsymbol{H}^{*}(T)-\boldsymbol{H}_{d}\right)=\left(\boldsymbol{E}^{*}(T)-\boldsymbol{E}_{d}, \boldsymbol{H}^{*}(T)-\boldsymbol{H}_{d}\right)$. Thus, the assertion is true.

\section{Semismooth Newton method For (P)}

We present a semismooth Newton method for the numerical solution of the optimal control problem $(\mathrm{P})$. Here, our main strategy is to reformulate the coupled optimality system (3.34) as an operator equation of the form

$$
\Psi\left(\boldsymbol{u}^{*}, a^{*}\right)=0 \quad \text { in } \boldsymbol{L}^{2}\left(\Omega_{c}\right) \times L^{2}(0, T) .
$$

To this aim, we consider a linear and bounded operator $\mathcal{R}: \boldsymbol{L}^{2}\left(\Omega_{c}\right) \rightarrow \boldsymbol{H}_{0}\left(\operatorname{curl} ; \Omega_{c}\right), \boldsymbol{y} \mapsto \boldsymbol{w}$, where $\boldsymbol{w} \in$ $\boldsymbol{H}_{0}\left(\operatorname{curl} ; \Omega_{c}\right)$ is the unique solution to the linear saddle point problem:

$$
\left\{\begin{aligned}
(\operatorname{curl} \boldsymbol{w}, \operatorname{curl} \boldsymbol{v})_{\boldsymbol{L}^{2}\left(\Omega_{c}\right)}+(\boldsymbol{v}, \nabla \varphi)_{\boldsymbol{L}^{2}\left(\Omega_{c}\right)} & =(\boldsymbol{y}, \boldsymbol{v})_{\boldsymbol{L}^{2}\left(\Omega_{c}\right)} & \forall \boldsymbol{v} \in \boldsymbol{H}_{0}\left(\operatorname{curl} ; \Omega_{c}\right), \\
(\boldsymbol{w}, \nabla \phi)_{\boldsymbol{L}^{2}\left(\Omega_{c}\right)} & =0 & \forall \phi \in H_{0}^{1}\left(\Omega_{c}\right) .
\end{aligned}\right.
$$

We note that the bilinear form $b: \boldsymbol{H}_{0}\left(\operatorname{curl} ; \Omega_{c}\right) \times H_{0}^{1}\left(\Omega_{c}\right) \rightarrow \mathbb{R}, b(\boldsymbol{w}, \phi)=(\boldsymbol{w}, \nabla \phi)_{\boldsymbol{L}^{2}\left(\Omega_{c}\right)}$, fulfills the Ladyzhenskaya-Babuška-Brezzi (LBB) condition:

$$
\begin{aligned}
\sup _{\boldsymbol{w} \in \boldsymbol{H}_{0}\left(\operatorname{curl} ; \Omega_{c}\right)} \frac{|b(\boldsymbol{w}, \phi)|}{\|\boldsymbol{w}\|_{\boldsymbol{H}\left(\mathbf{c u r l} ; \Omega_{c}\right)}} & \geq \frac{|b(\nabla \phi, \phi)|}{\|\nabla \phi\|_{\boldsymbol{H}\left(\mathbf{c u r l} ; \Omega_{c}\right)}} \\
& =\|\nabla \phi\|_{L^{2}\left(\Omega_{c}\right)} \geq c\|\phi\|_{H_{0}^{1}\left(\Omega_{c}\right)}, \quad \forall \phi \in H_{0}^{1}\left(\Omega_{c}\right),
\end{aligned}
$$

with a constant $c>0$, depending only on $\Omega_{c}$. In view of (4.2) and (3.10), the mixed variational problem (4.1) admits, for every $\boldsymbol{y} \in \boldsymbol{L}^{2}\left(\Omega_{c}\right)$, a unique solution $(\boldsymbol{w}, \varphi) \in \boldsymbol{H}_{0}\left(\operatorname{curl} ; \Omega_{c}\right) \times H_{0}^{1}\left(\Omega_{c}\right)$ satisfying

$$
\|\boldsymbol{w}\|_{\boldsymbol{H}\left(\mathbf{c u r l} ; \Omega_{c}\right)}+\|\varphi\|_{H_{0}^{1}\left(\Omega_{c}\right)} \leq c\|\boldsymbol{y}\|_{L^{2}\left(\Omega_{c}\right)}
$$

with a constant $c>0$, depending only on $\Omega_{c}$. This follows from a well-known existence and uniqueness result for linear saddle point problems [3].

Having introduced the linear and bounded operator $\mathcal{R}: \boldsymbol{L}^{2}\left(\Omega_{c}\right) \rightarrow \boldsymbol{H}_{0}\left(\mathbf{c u r l} ; \Omega_{c}\right)$, we now define the nonlinear operator $\Psi: \boldsymbol{L}^{2}\left(\Omega_{c}\right) \times L^{2}(0, T) \rightarrow \boldsymbol{L}^{2}\left(\Omega_{c}\right) \times L^{2}(0, T)$ as follows:

$$
\Psi(\boldsymbol{u}, a)=\left(\begin{array}{c}
\Psi_{1}(\boldsymbol{u}, a) \\
\Psi_{2}(\boldsymbol{u}, a)
\end{array}\right):=\left(\begin{array}{c}
\mathcal{R}\left(-\lambda_{\boldsymbol{u}}^{-1}(\boldsymbol{K}(\boldsymbol{u}, a), a)_{L^{2}(0, T)}\right)-\boldsymbol{u} \\
\mathbb{P}_{\left[a_{\min }, a_{\max }\right]}\left(-\lambda_{a}^{-1}(\boldsymbol{K}(\boldsymbol{u}, a), \boldsymbol{u})_{\boldsymbol{L}^{2}\left(\Omega_{c}\right)}\right)-a
\end{array}\right)
$$


where $\boldsymbol{K}(\boldsymbol{u}, a)=\boldsymbol{K} \in \mathcal{C}\left([0, T], \boldsymbol{L}^{2}(\Omega)\right)$ is given by

$$
(\boldsymbol{K}, \boldsymbol{Q})(t):=\mathbb{T}_{T-t}^{*}\left(\mathcal{S}(\boldsymbol{u}, a)-\left(\boldsymbol{E}_{d}, \boldsymbol{H}_{d}\right)\right) \quad \forall t \in[0, T] .
$$

According to the definition of $\Psi$, the optimality system (3.34) is equivalent to the following operator equation:

$$
\Psi\left(\boldsymbol{u}^{*}, a^{*}\right)=0 \quad \text { in } \boldsymbol{L}^{2}\left(\Omega_{c}\right) \times L^{2}(0, T)
$$

Therefore, the optimality system (3.34) can be solved by finding a root of the nonlinear operator $\Psi: \boldsymbol{L}^{2}\left(\Omega_{c}\right) \times$ $L^{2}(0, T) \rightarrow \boldsymbol{L}^{2}\left(\Omega_{c}\right) \times L^{2}(0, T)$.

Note that $\Psi_{1}: \boldsymbol{L}^{2}\left(\Omega_{c}\right) \times L^{2}(0, T) \rightarrow \boldsymbol{L}^{2}\left(\Omega_{c}\right)$ is continuously Fréchet-differentiable. However, due to the presence of the projection operator $\mathbb{P}_{\left[a_{\min }, a_{\max }\right]}: L^{2}(0, T) \rightarrow L^{2}(0, T)$, the operator $\Psi_{2}: \boldsymbol{L}^{2}\left(\Omega_{c}\right) \times L^{2}(0, T) \rightarrow$ $L^{2}(0, T)$ is not Fréchet-differentiable. Using the concept of Newton-semismoothness on $\Psi_{2}$, we may apply an altered Newton method, also known as the semismooth Newton method (see [10,27]) to solve (4.4). We recall the notion of Newton-semismoothness in the following definition:

Definition 4.1. Let $X, Y$ be Banach Spaces and $U$ an open subset of $X$. The mapping $F: U \rightarrow Y$ is called semismooth in $U$, if there exists a (possibly set-valued) mapping $\partial F: U \rightrightarrows L(X, Y)$ such that

$$
\sup _{G \in \partial F(x+\delta x)}\|F(x+\delta x)-F(x)-G \delta x\|_{Y}=o\left(\|\delta x\|_{X}\right) \quad \text { as }\|\delta x\|_{X} \rightarrow 0,
$$

for all $x \in U$. The set $\partial F$ and its elements are referred to as the Newton differential and Newtonian maps, respectively.

Let us introduce the operator

$$
\mathcal{G}: \boldsymbol{L}^{2}\left(\Omega_{c}\right) \times L^{2}(0, T) \rightarrow \mathcal{C}([0, T]), \quad(\boldsymbol{u}, a) \mapsto\left(-\lambda_{a}^{-1} \boldsymbol{K}(\boldsymbol{u}, a), \boldsymbol{u}\right)_{\boldsymbol{L}^{2}\left(\Omega_{c}\right)} .
$$

Employing this operator, $\Psi_{2}$ is equivalent to

$$
\Psi_{2}(\boldsymbol{u}, a)=\mathbb{P}_{\left[a_{\min }, a_{\max }\right]}(\mathcal{G}(\boldsymbol{u}, a))-a .
$$

It is well known that the projection operator $\mathbb{P}_{\left[a_{\min }, a_{\max }\right]}: L^{q}(0, T) \rightarrow L^{p}(0, T)$ is semismooth for $1 \leq p<q \leq \infty$ (see [10,27]). Furthermore, in the view of (4.3), the operator $\mathcal{G}: \boldsymbol{L}^{2}\left(\Omega_{c}\right) \times L^{2}(0, T) \rightarrow \mathcal{C}([0, T])$ is continuously Fréchet-differentiable. Therefore, choosing $p=2$ and $q=\infty$, it follows from $[10,27]$ that the operator $\Psi_{2}$ : $\boldsymbol{L}^{2}\left(\Omega_{c}\right) \times L^{2}(0, T) \rightarrow L^{2}(0, T)$ is semismooth.

The application of the corresponding Newton differential $\partial \Psi(\boldsymbol{u}, a)$ to the direction $(\delta \boldsymbol{u}, \delta a)$ is given by

$$
\begin{aligned}
& \partial \Psi(\boldsymbol{u}, a)(\delta \boldsymbol{u}, \delta a)=\left(\begin{array}{c}
\Psi_{1}^{\prime}(\boldsymbol{u}, a)(\delta \boldsymbol{u}, \delta a) \\
\partial \Psi_{2}(\boldsymbol{u}, a)(\delta \boldsymbol{u}, \delta a)
\end{array}\right) \\
& =\left(\begin{array}{c}
-\lambda_{\boldsymbol{u}}^{-1} \mathcal{R}\left(\left(\boldsymbol{K}^{\prime}(\boldsymbol{u}, a)(\delta \boldsymbol{u}, \delta a), a\right)_{L^{2}(0, T)}+(\boldsymbol{K}(\boldsymbol{u}, a), \delta a)_{L^{2}(0, T)}\right)-\delta \boldsymbol{u} \\
\mathcal{G}^{\prime}(\boldsymbol{u}, a)(\delta \boldsymbol{u}, \delta a) n(\boldsymbol{u}, a)-\delta a
\end{array}\right) \\
& =\left(\begin{array}{c}
-\lambda_{\boldsymbol{u}}^{-1} \mathcal{R}\left((\delta \boldsymbol{K}, a)_{L^{2}(0, T)}+(\boldsymbol{K}(\boldsymbol{u}, a), \delta a)_{L^{2}(0, T)}\right)-\delta \boldsymbol{u} \\
-\lambda_{a}^{-1}\left((\delta \boldsymbol{K}, \boldsymbol{u})_{\boldsymbol{L}^{2}\left(\Omega_{c}\right)}+(\boldsymbol{K}(\boldsymbol{u}, a), \delta \boldsymbol{u})_{\boldsymbol{L}^{2}\left(\Omega_{c}\right)}\right) n(\boldsymbol{u}, a)-\delta a
\end{array}\right),
\end{aligned}
$$


where

$$
n(\boldsymbol{u}, a)= \begin{cases}0, & \text { if } \mathcal{G}(\boldsymbol{u}, a) \notin\left(a_{\min }, a_{\max }\right), \\ 1, & \text { if } \mathcal{G}(\boldsymbol{u}, a) \in\left(a_{\min }, a_{\max }\right), \\ \xi, & \text { if } \mathcal{G}(\boldsymbol{u}, a) \in\left\{a_{\min }, a_{\max }\right\},\end{cases}
$$

with a fixed constant $\xi \in \mathbb{R}$. Here, $\boldsymbol{K}^{\prime}(\boldsymbol{u}, a)(\delta \boldsymbol{u}, \delta a)=\delta \boldsymbol{K}$ is given by

$$
(\delta \boldsymbol{K}, \delta \boldsymbol{Q})(t)=\mathbb{T}_{T-t}^{*}\left(\mathcal{S}^{\prime}(\boldsymbol{u}, a)(\delta \boldsymbol{u}, \delta a)\right) \quad \forall t \in[0, T],
$$

where

$$
\mathcal{S}^{\prime}(\boldsymbol{u}, a)(\delta \boldsymbol{u}, \delta a)=\int_{0}^{T} \mathbb{T}_{T-s}\left(\epsilon^{-1} \chi_{\Omega_{c}} \delta \boldsymbol{u} a(s)+\boldsymbol{u} \delta a(s), 0\right) \mathrm{d} s .
$$

In what follows, the constant $\xi \in \mathbb{R}$ in (4.6) is set to be zero. This choice leads to a standard active set method, as we shall explain shortly.

Algorithm 4.2 (Semismooth Newton Method for $(\mathrm{P})$ ).

1. Choose $\left(\boldsymbol{u}_{0}, a_{0}\right) \in \boldsymbol{L}^{2}\left(\Omega_{c}\right) \times L^{2}(0, T)$ and set $k=0$.

2. Compute $\Psi\left(\boldsymbol{u}_{k}, a_{k}\right)$. If $\Psi\left(\boldsymbol{u}_{k}, a_{k}\right)=0$, then stop.

3. Solve

$$
\partial \Psi\left(\boldsymbol{u}_{k}, a_{k}\right)\left(\delta \boldsymbol{u}_{k}, \delta a_{k}\right)=-\Psi\left(\boldsymbol{u}_{k}, a_{k}\right) .
$$

4. Set $\left(\boldsymbol{u}_{k+1}, a_{k+1}\right)=\left(\boldsymbol{u}_{k}, a_{k}\right)+\left(\delta \boldsymbol{u}_{k}, \delta a_{k}\right), \mathrm{k}=\mathrm{k}+1$, and return to 2 .

The stopping condition $\Psi\left(\boldsymbol{u}_{k}, a_{k}\right)=0$ of Algorithms 4.2 is only of theoretical importance. In our numerical practice, we stop the algorithm as soon as the norm $\left\|\Psi\left(\boldsymbol{u}_{k}, a_{k}\right)\right\|_{\boldsymbol{L}^{2}\left(\Omega_{c}\right) \times L^{2}(0, T)}$ drops below a userspecified stopping tolerance. The linear system (4.8) is solved by a conjugated gradient method. Note that, by the structure of the projection operator and its semismooth derivative, (4.8) can be reformulated as

$$
\begin{aligned}
& \left(\begin{array}{c}
\Psi_{1}^{\prime}\left(\boldsymbol{u}_{k}, a_{k}\right)\left(\delta \boldsymbol{u}_{k}, \delta a_{k}\right) \\
\mathcal{G}^{\prime}\left(\boldsymbol{u}_{k}, a_{k}\right)\left(\delta \boldsymbol{u}_{k}, \delta a_{k}\right) \chi_{\mathcal{I}_{k}}-\delta a_{k}
\end{array}\right)= \\
& \left(\begin{array}{c}
-\Psi_{1}\left(\boldsymbol{u}_{k}, a_{k}\right) \\
-\mathcal{G}\left(\boldsymbol{u}_{k}, a_{k}\right) \chi_{\mathcal{I}_{k}}-a_{\max } \chi_{\mathcal{A}_{k}^{+}}-a_{\min } \chi_{\mathcal{A}_{k}^{-}}+a_{k}
\end{array}\right),
\end{aligned}
$$

where we use the following partition of $(0, T)$ into the active and inactive sets $\mathcal{A}_{k}=\mathcal{A}_{k}^{-} \cup \mathcal{A}_{k}^{+}$and $\mathcal{I}_{k}$ :

$$
\begin{aligned}
\mathcal{A}_{k}^{-} & :=\left\{t \in(0, T): \mathcal{G}\left(\boldsymbol{u}_{k}, a_{k}\right)<a_{\min }\right\} \\
\mathcal{A}_{k}^{+} & :=\left\{t \in(0, T): \mathcal{G}\left(\boldsymbol{u}_{k}, a_{k}\right)>a_{\max }\right\} \\
\mathcal{I}_{k} & :=(0, T) \backslash \mathcal{A}_{k} .
\end{aligned}
$$

Remark 4.3. The vector field $\boldsymbol{K}(\boldsymbol{u}, a)=\boldsymbol{K}$ in (4.3) is obtained by solving the following coupled partial differential equations:

$$
\begin{aligned}
& \begin{cases}\epsilon \boldsymbol{E}_{t}-\operatorname{curl} \boldsymbol{H}+\sigma \boldsymbol{E}=\chi_{\Omega_{c}} \boldsymbol{u} a & \text { in } \Omega \times(0, T), \\
\mu \boldsymbol{H}_{t}+\operatorname{curl} \boldsymbol{E}=0 & \text { in } \Omega \times(0, T), \\
\boldsymbol{E} \times \boldsymbol{n}=0 & \text { in } \Gamma \times(0, T), \\
\boldsymbol{E}(0)=\boldsymbol{E}_{0} & \text { in } \Omega, \\
\boldsymbol{H}(0)=\boldsymbol{H}_{0} & \text { in } \Omega,\end{cases} \\
& \begin{cases}\epsilon \boldsymbol{K}_{t}-\operatorname{curl} \boldsymbol{Q}-\sigma \boldsymbol{K}=0 & \text { in } \Omega \times(0, T), \\
\mu \boldsymbol{Q}_{t}+\operatorname{curl} \boldsymbol{K}=0 & \text { in } \Omega \times(0, T), \\
\boldsymbol{K} \times \boldsymbol{n}=0 & \text { in } \Gamma \times(0, T), \\
\boldsymbol{K}(T)=\boldsymbol{E}(T)-\boldsymbol{E}_{d} & \text { in } \Omega, \\
\boldsymbol{Q}(T)=\boldsymbol{H}(T)-\boldsymbol{H}_{d} & \text { in } \Omega .\end{cases}
\end{aligned}
$$


Furthermore, by definition, $\boldsymbol{w}=\mathcal{R}\left(-\lambda_{\boldsymbol{u}}^{-1}(\boldsymbol{K}, a)_{L^{2}(0, T)}\right)$ is the weak solution to

$$
\left\{\begin{aligned}
\operatorname{curl} \operatorname{curl} \boldsymbol{w}+\nabla \varphi & =-\lambda_{\boldsymbol{u}}^{-1}(\boldsymbol{K}, a)_{L^{2}(0, T)} & & \text { in } \Omega_{c}, \\
\operatorname{div} \boldsymbol{w} & =0 & & \text { in } \Omega_{c}, \\
\boldsymbol{w} \times \boldsymbol{n}_{c} & =0 & & \text { on } \Gamma_{c} .
\end{aligned}\right.
$$

In conclusion, to compute $\Psi(\boldsymbol{u}, a)$, we have to solve (4.10a)-(4.10b) and (4.11). Also, let us remark that $\boldsymbol{K}^{\prime}(\boldsymbol{u}, a)(\delta \boldsymbol{u}, \delta a)=\delta \boldsymbol{K}$ in (4.7) is calculated by solving the following coupled partial differential equations:

$$
\begin{aligned}
& \begin{cases}\epsilon \delta \boldsymbol{E}_{t}-\operatorname{curl} \delta \boldsymbol{H}+\sigma \delta \boldsymbol{E}=\chi_{\Omega_{c}}(\delta \boldsymbol{u} a+\boldsymbol{u} \delta a) & \text { in } \Omega \times(0, T), \\
\mu \delta \boldsymbol{H}_{t}+\operatorname{curl} \delta \boldsymbol{E}=0 & \text { in } \Omega \times(0, T), \\
\delta \boldsymbol{E} \times \boldsymbol{n}=0 & \text { in } \Gamma \times(0, T), \\
\delta \boldsymbol{E}(0)=0 & \text { in } \Omega, \\
\delta \boldsymbol{H}(0)=0 & \text { in } \Omega,\end{cases} \\
& \begin{cases}\epsilon \delta \boldsymbol{K}_{t}-\operatorname{curl} \delta \boldsymbol{Q}-\sigma \delta \boldsymbol{K}=0 & \text { in } \Omega \times(0, T), \\
\mu \delta \boldsymbol{Q}_{t}+\operatorname{curl} \delta \boldsymbol{K}=0 & \text { in } \Omega \times(0, T), \\
\delta \boldsymbol{K} \times \boldsymbol{n}=0 & \text { in } \Gamma \times(0, T), \\
\delta \boldsymbol{K}(T)=\delta \boldsymbol{E}(T) & \text { in } \Omega, \\
\delta \boldsymbol{Q}(T)=\delta \boldsymbol{H}(T) & \text { in } \Omega .\end{cases}
\end{aligned}
$$

Note that, in the context of optimality system, (4.12a) and (4.12b) are also called the linearized state equation and the dual equation for Hessian, respectively. After calculating $\delta \boldsymbol{K}$, we obtain $\delta \boldsymbol{w}:=-\lambda_{\boldsymbol{u}}^{-1} \mathcal{R}\left((\delta \boldsymbol{K}, a)_{L^{2}(0, T)}+\right.$ $\left.(\boldsymbol{K}(\boldsymbol{u}, a), \delta a)_{L^{2}(0, T)}\right)$ by solving

$$
\left\{\begin{aligned}
\operatorname{curl} \operatorname{curl} \delta \boldsymbol{w}+\nabla \varphi & =-\lambda_{\boldsymbol{u}}^{-1}\left((\delta \boldsymbol{K}, a)_{L^{2}(0, T)}+(\boldsymbol{K}, \delta a)_{L^{2}(0, T)}\right) & & \text { in } \Omega_{c}, \\
\operatorname{div} \delta \boldsymbol{w} & =0 & & \text { in } \Omega_{c} \\
\delta \boldsymbol{w} \times \boldsymbol{n}_{c} & =0 & & \text { on } \Gamma_{c} .
\end{aligned}\right.
$$

In conclusion, to compute $\partial \Psi(\boldsymbol{u}, a)(\delta \boldsymbol{u}, \delta a)$, we have to solve (4.12a)-(4.12b) and (4.13). Note that $\varphi$ in the equations (4.11) and (4.13) are different functions.

\section{Numerical EXAMPLES}

In this section, numerical examples are presented to illustrate the previously discussed semismooth Newton method. As pointed out in Remark 4.3, for the calculation of $\Psi(\boldsymbol{u}, a)$ and $\partial \Psi(\boldsymbol{u}, a)(\delta \boldsymbol{u}, \delta a)$ in Algorithm 4.2, three different types of PDEs emerge: Forward Maxwell's equations (4.10a), (4.12a), backward Maxwell's equations (4.10b), (4.12b), and magnetostatic equations (4.11), (4.13). Their discretization is carried out by employing the mixed method by Monk [18] ( $c f$. also [4]) for the full Maxwell equations (forward and backward) and a well-known mixed method for the magnetostatic equations. More precisely, we use the lowest order edge elements of Nédélec's first family for the magnetic field $\boldsymbol{H}$ along with piecewise constant vector functions for the electric field $\boldsymbol{E}$. Furthermore, time discretization is done by the Crank-Nicolson (CN) scheme as in [18]. The stability analysis for the fully discrete formulation is described in [18]. Hereby, a positive semi-definite $\sigma$ is key for proving a stability estimate of the time steps of the CN scheme by the initial value. All results from [18] can also be applied to the backward Maxwell equations for $\boldsymbol{Q}$ and $\boldsymbol{K}$, as the flipped sign in front of $\sigma$ leads to an estimation by the final time. Furthermore, we discretize the mixed magnetostatic equations (3.34c) and (4.13) by utilizing the lowest order edge elements of Nédélec's first family for $\boldsymbol{w}$ (or $\partial \boldsymbol{w}$ ) and continuous piecewise linear elements for $\varphi$. For this choice of mixed elements, the discrete LBB condition is satisfied.

We use the FEniCS library [17] for the realization of the finite element discretization in space. Time stepping and the semismooth Newton algorithm including the conjugate gradient method for solving every Newton 
TABlE 1. First example: Convergence history.

\begin{tabular}{ccc}
\hline Step & Error & \# cg-it. \\
\hline 1 & $2.8876 \times 10^{0}$ & 9 \\
2 & $1.2168 \times 10^{0}$ & 10 \\
3 & $6.0533 \times 10^{-1}$ & 7 \\
4 & $3.3776 \times 10^{-1}$ & 7 \\
5 & $1.6456 \times 10^{-1}$ & 7 \\
6 & $8.2886 \times 10^{-2}$ & 7 \\
7 & $4.0255 \times 10^{-2}$ & 7 \\
\hline
\end{tabular}

\begin{tabular}{ccc}
\hline Step & Error & \# cg-it. \\
\hline 8 & $1.9716 \times 10^{-2}$ & 7 \\
9 & $9.6272 \times 10^{-3}$ & 7 \\
10 & $4.7086 \times 10^{-3}$ & 7 \\
11 & $2.3012 \times 10^{-3}$ & 7 \\
12 & $1.1253 \times 10^{-3}$ & 7 \\
13 & $5.4999 \times 10^{-4}$ & 7 \\
\hline
\end{tabular}

step (4.9) are manually programmed via the python interface of FEniCS. The iterations for the algorithm are stopped if the active sets remain the same and

$$
\left(\left\|\Psi_{1}(\boldsymbol{u}, a)\right\|_{L^{2}\left(\Omega_{c}\right)}^{2}+\left\|\Psi_{2}(\boldsymbol{u}, a)\right\|_{L^{2}(0, T)}^{2}\right)^{\frac{1}{2}}<\delta
$$

is fulfilled with $\delta=10^{-3}$. Moreover, the conjugated gradient method is terminated when the corresponding residual drops below $10^{-6}$.

\subsection{First example}

Let us consider the computational domain

$$
\Omega=\Omega_{c}=(0,1) \times(0,1) \times(0,0.5),
$$

and the time domain $(0,1)$. For the spatial discretization, we employ a regular tetrahedral mesh with the meshsize $h=2^{-5}$. This leads to 415824 degrees of freedom. Furthermore, the time interval is discretized uniformly with the step-size $k=2^{-5}$.

The material functions are set to be constant $\epsilon=10, \mu=1$ and $\sigma=0$. Furthermore, we choose $\lambda_{a}=\lambda_{u}=0.05$, $a_{\min }=4$, and $a_{\max }=9.5$. The desired and initial electromagnetic fields are given by

$$
\boldsymbol{E}_{d}=\left(\begin{array}{c}
0 \\
0 \\
\sin (2 \pi x) \sin (2 \pi y)
\end{array}\right), \quad \boldsymbol{H}_{d}=2 \pi\left(\begin{array}{c}
-\sin (2 \pi x) \cos (2 \pi y) \\
\cos (2 \pi x) \sin (2 \pi y) \\
0
\end{array}\right)
$$

and

$$
\boldsymbol{E}_{0}=\left(\begin{array}{l}
0 \\
0 \\
0
\end{array}\right), \quad \boldsymbol{H}_{0}=\frac{1}{10}\left(\begin{array}{c}
-\sin (\pi x) \cos (\pi y) \\
\cos (\pi x) \sin (\pi y) \\
0
\end{array}\right),
$$

where $\boldsymbol{H}_{0}$ and $\boldsymbol{H}_{d}$ are depicted in Figure 1.

The algorithm is initialized with the constant function $a_{0}=4$ and the vector field $\boldsymbol{u}_{0}=\boldsymbol{E}_{d}$. We note that 13 Newton iterations are required for successful termination of the algorithm. See Table 1 for the corresponding convergence history including the residual error and the number of conjugate gradient iterations for each Newton step. Moreover, Figures 2 and 3 show the computed optimal current density $\boldsymbol{u}_{h k}^{*}$ and the computed optimal amplitude $a_{h k}^{*}$. Lastly, the evolution of the computed optimal magnetic field $\boldsymbol{H}_{h k}^{*}$ is depicted in Figure 4. From the initial time $t=0$ on, a gradual change of $\boldsymbol{H}_{h k}^{*}$ is observed. At the final time $t=1$, the computed optimal magnetic field is close to the desired one. 


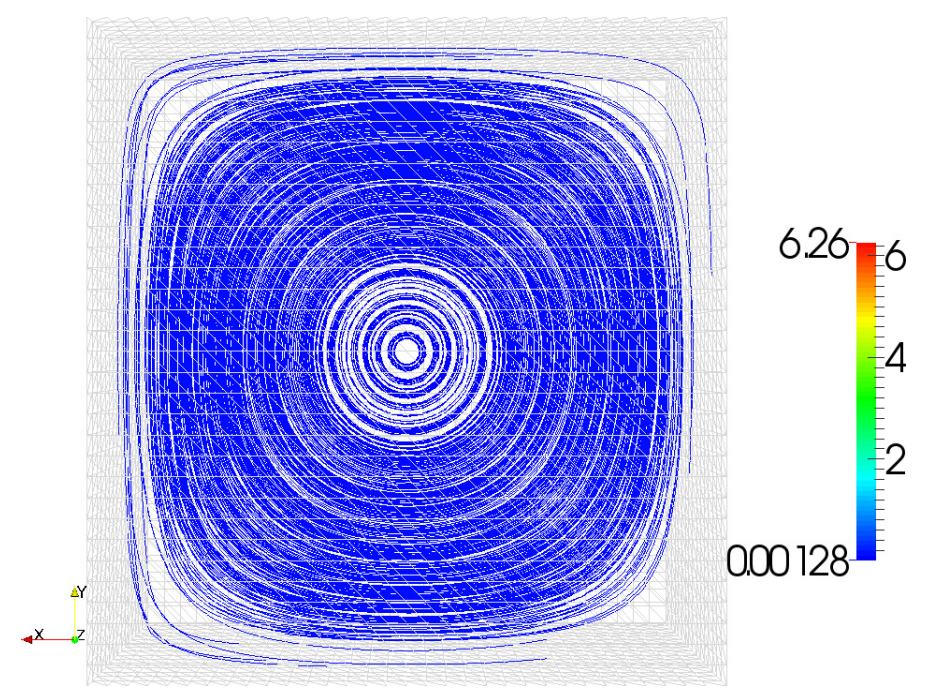

(a) $\boldsymbol{H}_{0}$

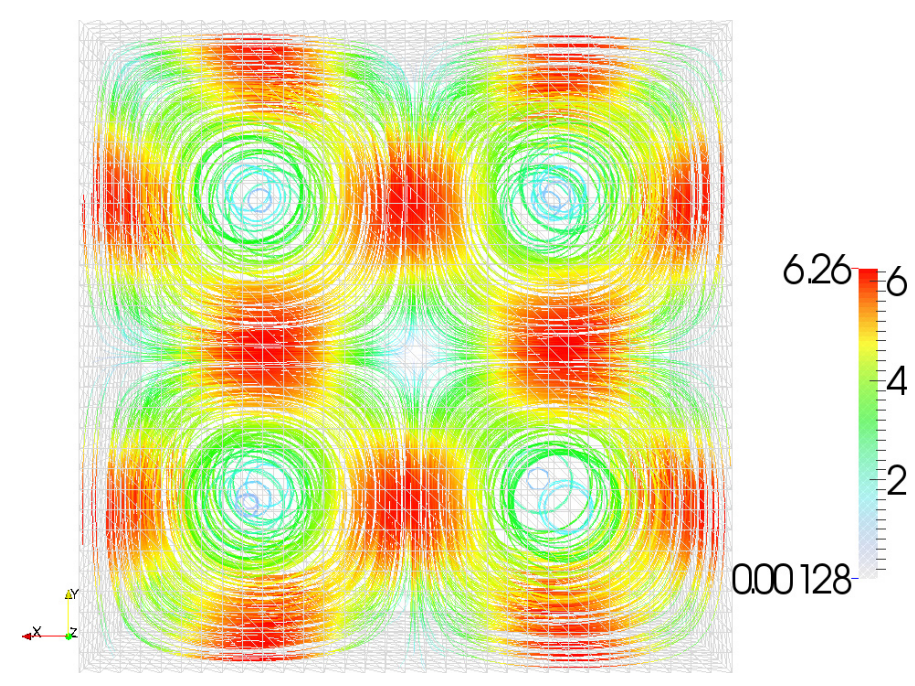

(b) $\boldsymbol{H}_{d}$

Figure 1. First example: Initial field $\boldsymbol{H}_{0}$ and desired magnetic field $\boldsymbol{H}_{d}$.

\subsection{Second example}

For the second example, let us consider the following L-shaped computational domain:

$$
\Omega=\Omega_{c}=(-0.5,0.5) \times(-0.5,0.5) \times(0,0.5) \backslash[0,0.5]^{3}
$$

and the time domain $(0,1)$. For the spatial discretization, we use a regular tetrahedral mesh with the mesh-size $h=2^{-5}$, which leads to 312656 degrees of freedom. Also, the time interval is discretized uniformly with the 


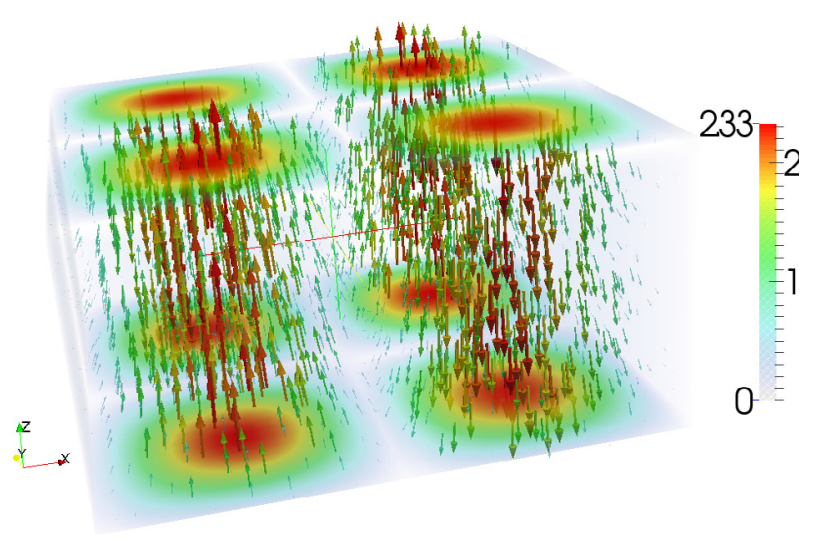

FiguRE 2. First example: Computed optimal current density $\boldsymbol{u}_{h k}^{*}$.

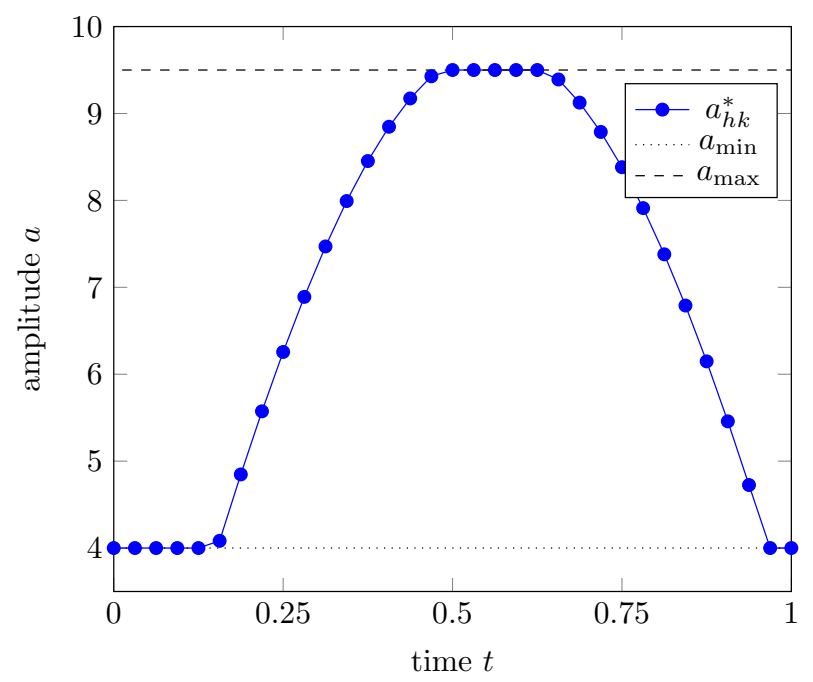

Figure 3. First example: Computed optimal amplitude $a_{h k}^{*}$.

step-size $k=2^{-5}$. We define the subdomains

$$
\begin{aligned}
& \Omega_{1}:=(-0.5,0) \times(0,0.5) \times(0,0.5), \\
& \Omega_{2}:=(-0.5,0) \times(-0.5,0) \times(0,0.5), \\
& \Omega_{3}:=(0,0.5) \times(-0.5,0) \times(0,0.5) .
\end{aligned}
$$

In contrast to the first example, the material functions are now set to be piecewise constant:

$$
\begin{aligned}
\epsilon & =1+0.5 \chi_{\Omega_{1}}+\chi_{\Omega_{3}}, \\
\sigma & =3 \chi_{\Omega_{2}}+2, \\
\mu & =0.5+\chi_{\Omega_{1}}+0.5 \chi_{\Omega_{3}},
\end{aligned}
$$




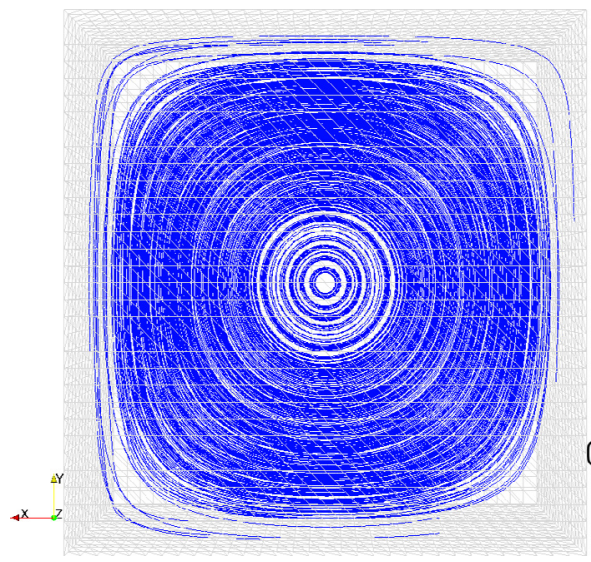

(a) $t=0$

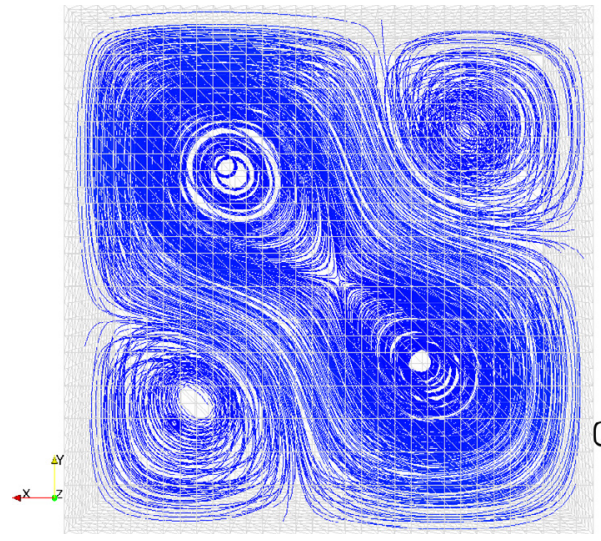

(c) $t=0.25$

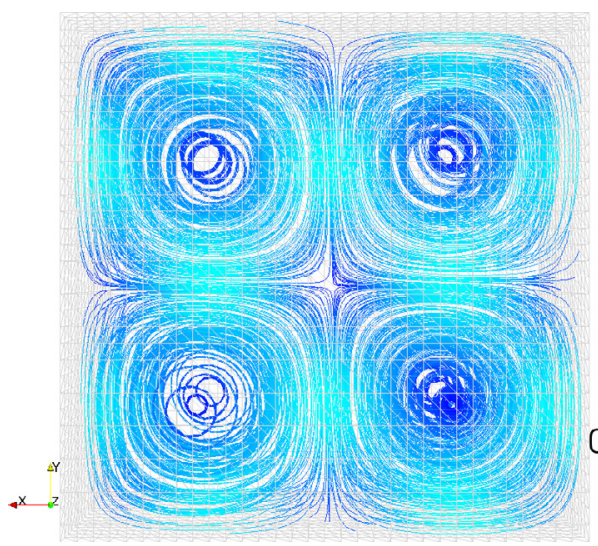

(e) $t=0.6875$

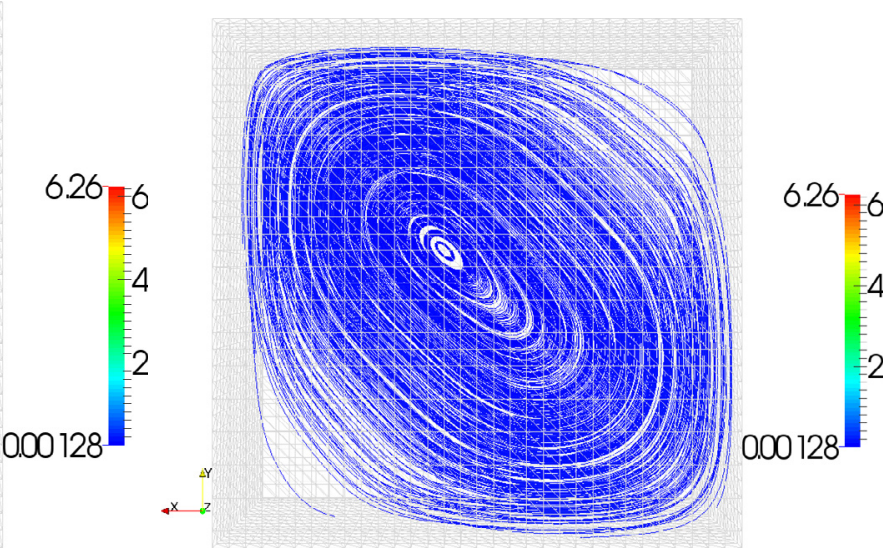

(b) $t=0.125$

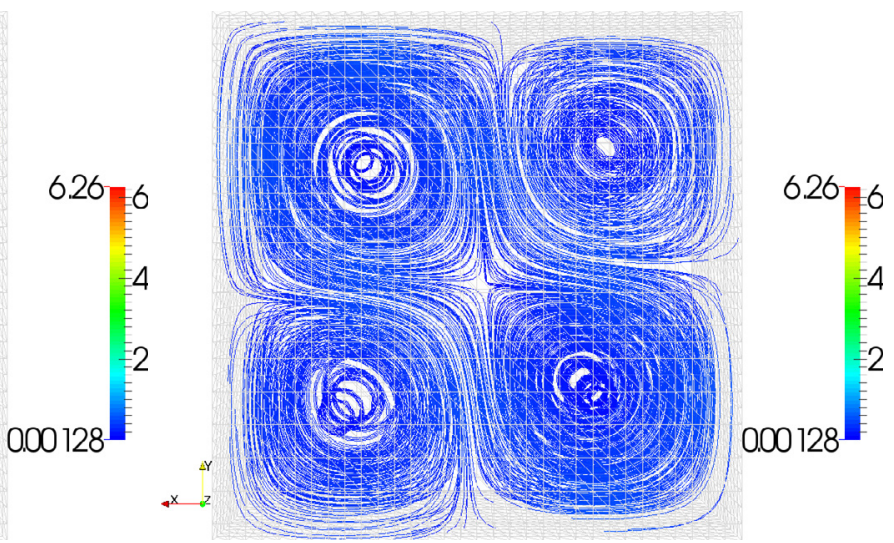

(d) $t=0.375$

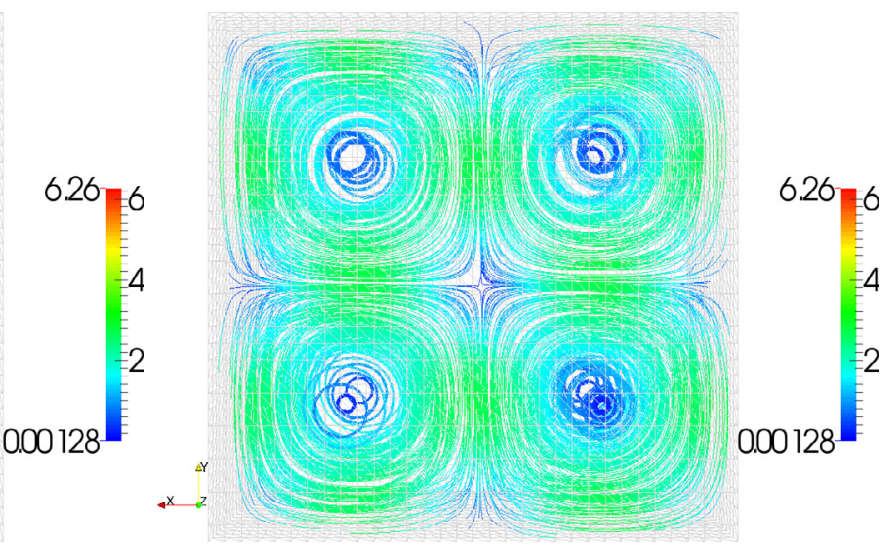

(f) $t=1$

Figure 4. First example: Evolution of the computed optimal magnetic field $\boldsymbol{H}_{h k}^{*}$. 


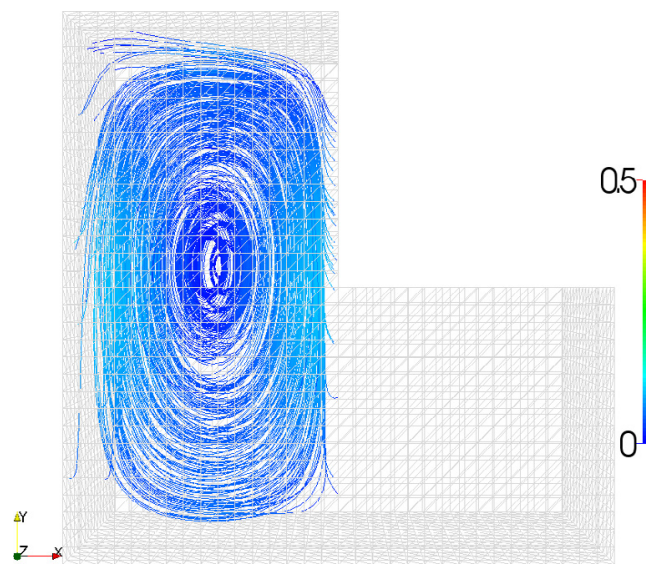

(a) $\boldsymbol{H}_{0}$

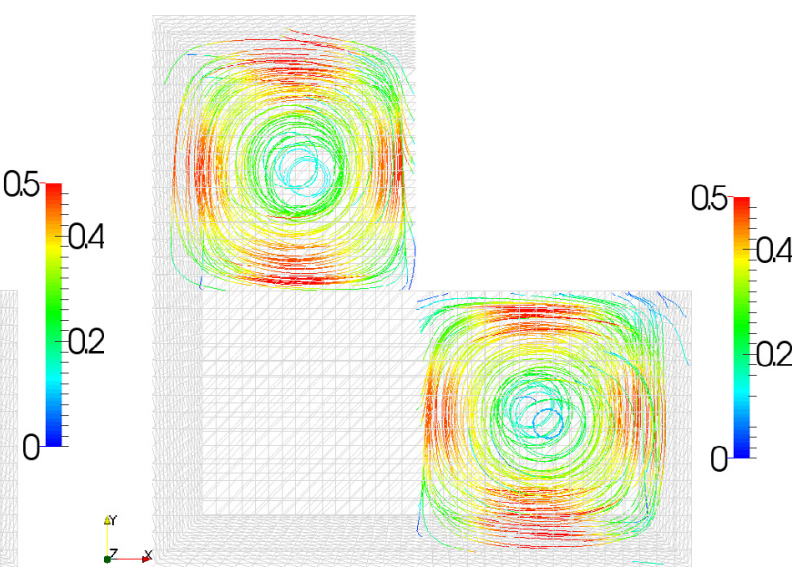

(b) $\boldsymbol{H}_{d}$

Figure 5. Second example: Initial field $\boldsymbol{H}_{0}$ and desired magnetic field $\boldsymbol{H}_{d}$.

TABle 2. Second example: Convergence history.

\begin{tabular}{ccc}
\hline Step & Error & \# cg-it. \\
\hline 1 & $6.0484 \times 10^{-2}$ & 4 \\
2 & $8.9691 \times 10^{-2}$ & 15 \\
3 & $1.0686 \times 10^{-1}$ & 18 \\
4 & $1.1067 \times 10^{-1}$ & 20 \\
5 & $9.2020 \times 10^{-2}$ & 24 \\
6 & $5.8430 \times 10^{-2}$ & 27 \\
\hline
\end{tabular}

\begin{tabular}{ccc}
\hline Step & Error & \# cg-it. \\
\hline 7 & $2.9691 \times 10^{-2}$ & 27 \\
8 & $1.3757 \times 10^{-2}$ & 23 \\
9 & $6.3001 \times 10^{-3}$ & 19 \\
10 & $2.8720 \times 10^{-3}$ & 15 \\
11 & $1.3248 \times 10^{-3}$ & 11 \\
12 & $6.0175 \times 10^{-4}$ & 9 \\
\hline
\end{tabular}

where $\chi_{\Omega_{i}}$ denotes the indicator function of the subdomain $\Omega_{i}$ with $i=1,2,3$. We consider the desired electric and magnetic fields

$$
\begin{gathered}
\boldsymbol{E}_{d}=\frac{\left(\chi_{\Omega_{1}}+\chi_{\Omega_{3}}\right)}{4 \pi}\left(\begin{array}{c}
0 \\
0 \\
\sin (2 \pi x) \sin (2 \pi y)
\end{array}\right), \\
\boldsymbol{H}_{d}=\frac{\left(\chi_{\Omega_{1}}+\chi_{\Omega_{3}}\right)}{2}\left(\begin{array}{c}
-\sin (2 \pi x) \cos (2 \pi y) \\
\cos (2 \pi x) \sin (2 \pi y) \\
0
\end{array}\right),
\end{gathered}
$$

and initial data

$$
\boldsymbol{E}_{0}=\left(\begin{array}{l}
0 \\
0 \\
0
\end{array}\right) \quad \text { and } \quad \boldsymbol{H}_{0}=\frac{\left(\chi_{\Omega_{1}}+\chi_{\Omega_{2}}\right)}{100}\left(\begin{array}{c}
5 \sin (\pi x) \cos (\pi y) \\
10 \cos (\pi x) \sin (\pi y) \\
0
\end{array}\right) .
$$

Furthermore, the Tikhonov parameters are $\lambda_{a}=\lambda_{u}=0.006$, and the lower and upper bounds are given by $a_{\min }=0$ and $a_{\max }=2$.

The algorithm is initialized with the constant function $a_{0}=1$ and the vector field $\boldsymbol{u}_{0}=\boldsymbol{E}_{d}$. We provide a detailed insight into the convergence behavior of the algorithm in Table 2, where for each of the 12 Newton iterations the number of cg-iterations and the corresponding residuals are shown.

Note that the initial magnetic field $\boldsymbol{H}_{0}$ acts only in the subdomains $\Omega_{1}$ and $\Omega_{2}$, whereas the desired magnetic field $\boldsymbol{H}_{d}$ is concentrated in $\Omega_{1}$ and $\Omega_{3}$ (see Fig. 5). Due to the structure of $\boldsymbol{H}_{d}$, it makes sense for the optimal 


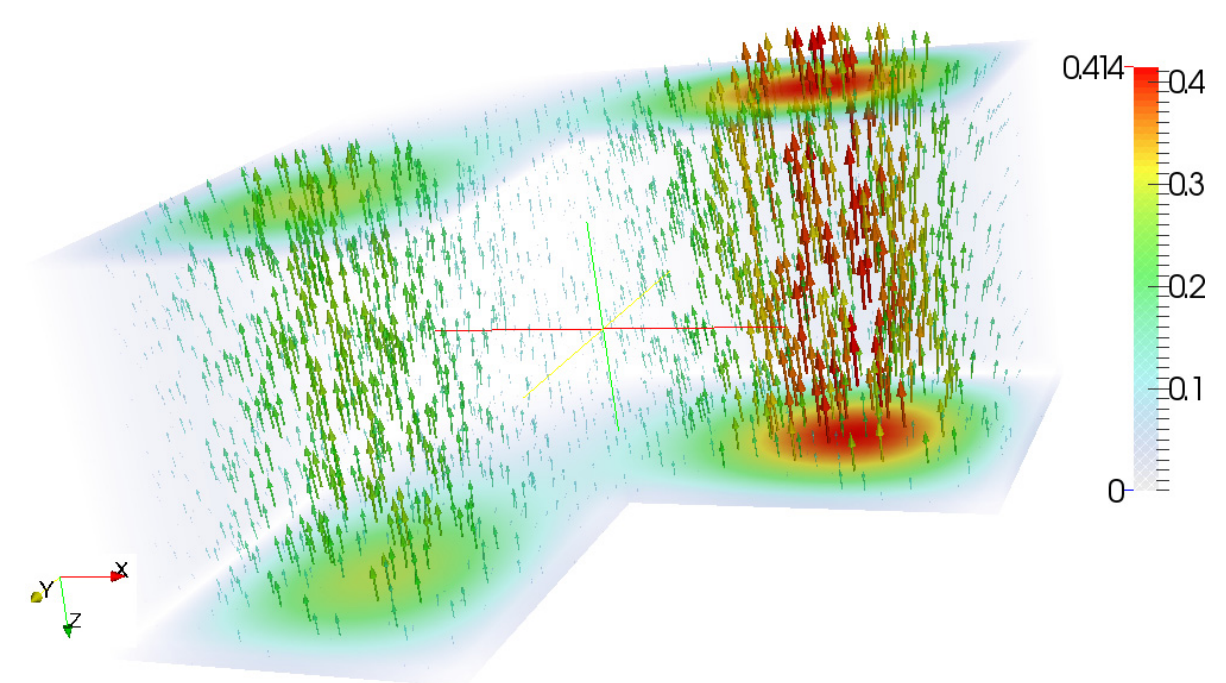

Figure 6. Second example: Computed optimal current density $\boldsymbol{u}_{h k}^{*}$.

current density to concentrate in the subdomains $\Omega_{1}$ and $\Omega_{3}$ to achieve the desired field $\boldsymbol{H}_{d}$. Indeed, the computed optimal current density $\boldsymbol{u}_{h k}^{*}$ features this behavior, as we can observe in Figure 6.

Figure 7 depicts the evolution of the computed optimal magnetic field $\boldsymbol{H}_{h k}^{*}$. In contrast to the first example, the computed solution exhibits a turbulent behavior. A possible reason for this behavior is due to the L-shaped structure of the domain and the jump discontinuities of the material functions, which lead to singularities in the solution of Maxwell's equations ( $c f$. Costabel et al. $[6,7]$ ). Nonetheless, at the final time $t=1$, the structure of $\boldsymbol{H}_{h k}^{*}$ reasonably approximates the desired one.

Remark 5.1. The choice of $\delta=10^{-3}$ for the stopping condition is simply justified by the fact that $h$ and $k$ are moderately chosen. In this case, a smaller residual gave similar results. Also, notice that we did not use smaller numbers for $h$ and $k$, as the computation of these examples is already highly expensive.

Under higher regularity assumptions, the mixed method by Monk [18] for the state approximation converges with error $\mathcal{O}\left(k^{2}+h\right)$. Furthermore, it is well known that the mixed method for the magnetostatic equation (3.34c) converges with a rate of $\delta_{c} \in(0,0.5]$ depending on the regularity of the control domain $\Omega_{c}$. This would be the precision one could expect for the numerical results of the optimal control problem. The a priori error analysis is a goal of our ongoing project.

\section{APPENDIX}

Lemma A.1. If $\xi \in \mathcal{C}^{0,1}\left(\overline{\Omega_{c}}\right)$ and $\boldsymbol{u} \in \boldsymbol{H}_{0}\left(\operatorname{curl} ; \Omega_{c}\right)$, then $\xi \boldsymbol{u} \in \boldsymbol{H}_{0}\left(\operatorname{curl} ; \Omega_{c}\right)$.

Proof. Let $\xi \in \mathcal{C}^{0,1}\left(\overline{\Omega_{c}}\right)$ and $\boldsymbol{u} \in \boldsymbol{H}_{0}\left(\mathbf{c u r l} ; \Omega_{c}\right)$. By the distributional definition of the curl-operator and using the well-known product rule for Sobolev functions, it holds that

$$
\xi \boldsymbol{u} \in \boldsymbol{H}\left(\operatorname{curl} ; \Omega_{c}\right) \text { and } \operatorname{curl}(\xi \boldsymbol{u})=\nabla \xi \times \boldsymbol{u}+\xi \operatorname{curl} \boldsymbol{u} .
$$

We denote now the tangential trace operator on $\Gamma_{c}$ by $\boldsymbol{\gamma}_{t}: \boldsymbol{H}\left(\mathbf{c u r l} ; \Omega_{c}\right) \rightarrow \boldsymbol{H}^{-\frac{1}{2}}\left(\Gamma_{c}\right)(c f .[2])$ satisfying

$$
\begin{array}{r}
\left\langle\gamma_{t}(\boldsymbol{u}), \boldsymbol{\tau}(\boldsymbol{\psi})\right\rangle_{\boldsymbol{H}^{-\frac{1}{2}}\left(\Gamma_{c}\right), \boldsymbol{H}^{\frac{1}{2}}\left(\Gamma_{c}\right)}=\int_{\Omega_{c}} \boldsymbol{u} \cdot \operatorname{curl} \boldsymbol{\psi} \mathrm{d} x-\int_{\Omega_{c}} \operatorname{curl} \boldsymbol{u} \cdot \boldsymbol{\psi} \mathrm{d} x \\
\forall \boldsymbol{u} \in \boldsymbol{H}\left(\operatorname{curl} ; \Omega_{c}\right), \boldsymbol{\psi} \in \boldsymbol{H}^{1}\left(\Omega_{c}\right),
\end{array}
$$




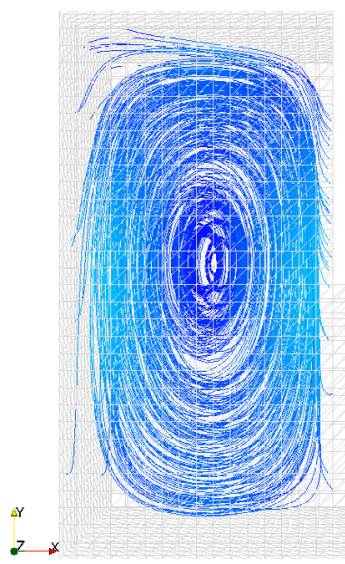

(a) $t=0$

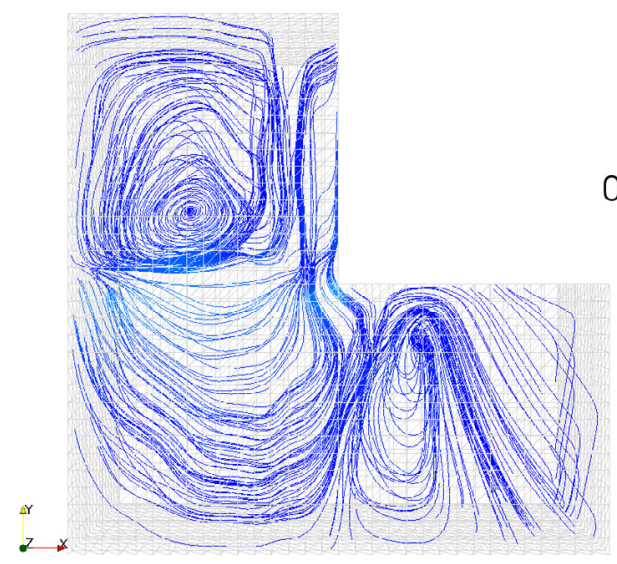

(c) $t=0.375$

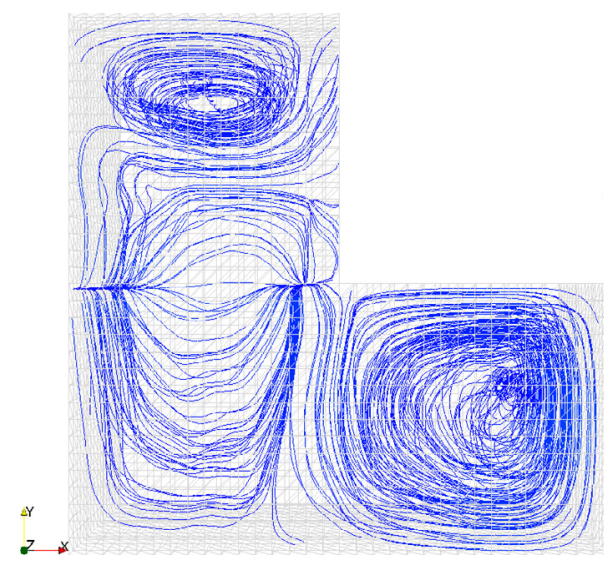

(e) $t=0.75$

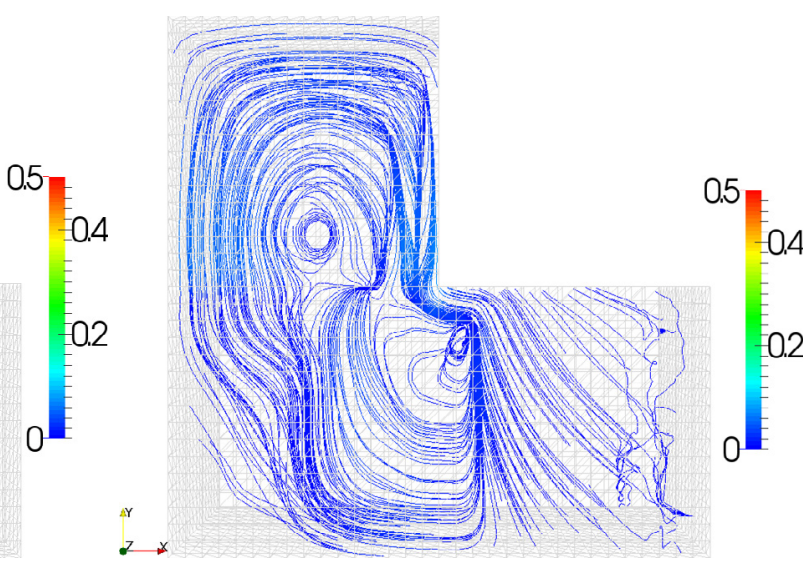

(b) $t=0.1875$

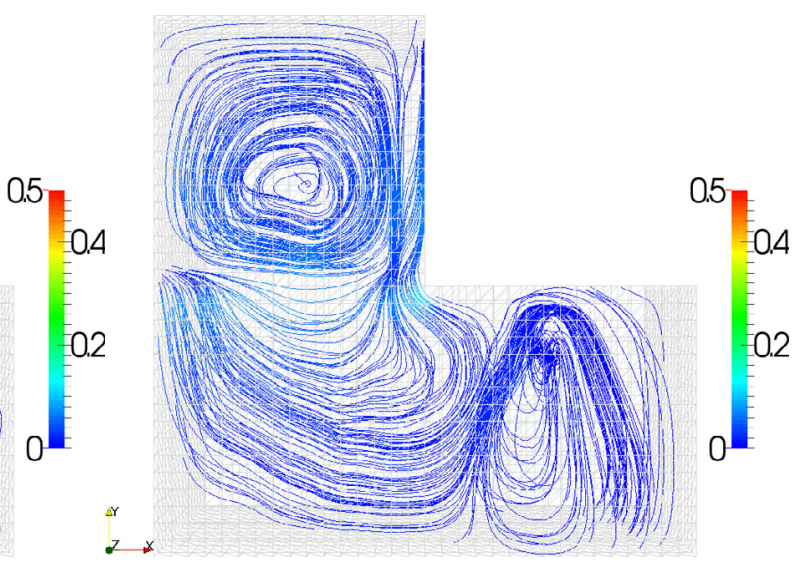

(d) $t=0.5$

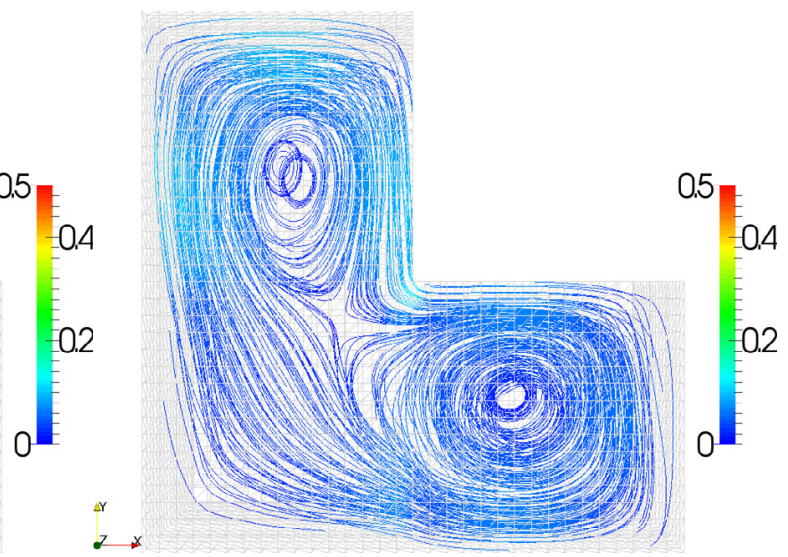

(f) $t=1$

FiguRE 7. Second example: Evolution of the computed optimal magnetic field $\boldsymbol{H}_{h k}^{*}$. 


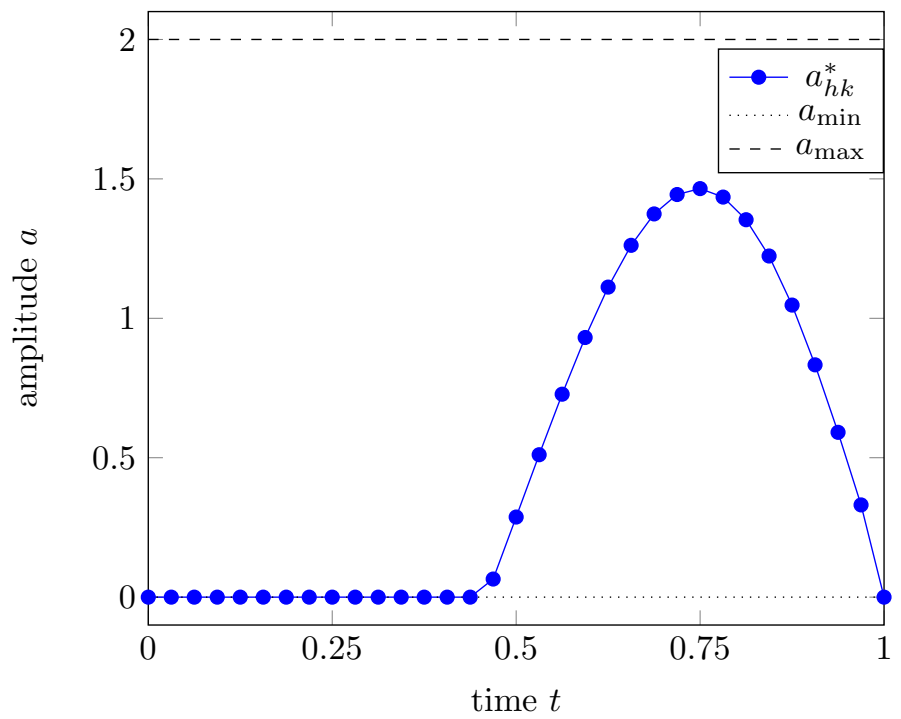

Figure 8. Second example: Computed optimal amplitude $a_{h k}^{*}$.

where $\boldsymbol{\tau}: \boldsymbol{H}^{1}\left(\Omega_{c}\right) \rightarrow \boldsymbol{H}^{\frac{1}{2}}\left(\Gamma_{c}\right)$ denotes the trace operator. Let us prove $\boldsymbol{\gamma}_{t}(\xi \boldsymbol{u})=0$. According to (A.2), it holds, for every $\boldsymbol{\psi} \in \boldsymbol{H}^{1}\left(\Omega_{c}\right)$, that

$$
\begin{aligned}
& \left\langle\gamma_{t}(\xi \boldsymbol{u}), \boldsymbol{\tau}(\boldsymbol{\psi})\right\rangle_{\boldsymbol{H}^{-\frac{1}{2}}\left(\Gamma_{c}\right), \boldsymbol{H}^{\frac{1}{2}}\left(\Gamma_{c}\right)}=\int_{\Omega_{c}} \xi \boldsymbol{u} \cdot \operatorname{curl} \boldsymbol{\psi} \mathrm{d} x-\int_{\Omega_{c}} \operatorname{curl}(\xi \boldsymbol{u}) \cdot \boldsymbol{\psi} \mathrm{d} x \\
& \underbrace{=}_{(\text {A.1) }} \int_{\Omega_{c}} \xi \boldsymbol{u} \cdot \operatorname{curl} \boldsymbol{\psi} \mathrm{d} x-\int_{\Omega_{c}}(\nabla \xi \times \boldsymbol{u}+\xi \operatorname{curl} \boldsymbol{u}) \cdot \boldsymbol{\psi} \mathrm{d} x \\
& \underbrace{=}_{\gamma_{t}(\boldsymbol{u})=0} \int_{\Omega_{c}} \xi \boldsymbol{u} \cdot \operatorname{curl} \boldsymbol{\psi} \mathrm{d} x-\int_{\Omega_{c}}(\nabla \xi \times \boldsymbol{u}) \cdot \boldsymbol{\psi}+\boldsymbol{u} \cdot \operatorname{curl}(\xi \boldsymbol{\psi}) \mathrm{d} x \\
& =\int_{\Omega_{c}} \xi \boldsymbol{u} \cdot \operatorname{curl} \boldsymbol{\psi} \mathrm{d} x-\int_{\Omega_{c}}(\nabla \xi \times \boldsymbol{u}) \cdot \boldsymbol{\psi}+\boldsymbol{u} \cdot(\nabla \xi \times \boldsymbol{\psi}+\xi \operatorname{curl} \boldsymbol{\psi}) \mathrm{d} x \\
& =-\int_{\Omega_{c}}(\nabla \xi \times \boldsymbol{u}) \cdot \boldsymbol{\psi}+\boldsymbol{u} \cdot(\nabla \xi \times \boldsymbol{\psi}) \mathrm{d} x .
\end{aligned}
$$

Finally, using the identities $\boldsymbol{a} \times \boldsymbol{b}=-\boldsymbol{b} \times \boldsymbol{a}$ and $\boldsymbol{a} \cdot(\boldsymbol{b} \times \boldsymbol{c})=(\boldsymbol{a} \times \boldsymbol{b}) \cdot \boldsymbol{c}$, we arrive at

$$
\left\langle\gamma_{t}(\xi \boldsymbol{u}), \boldsymbol{\tau}(\boldsymbol{\psi})\right\rangle_{\boldsymbol{H}^{-\frac{1}{2}}\left(\Gamma_{c}\right), \boldsymbol{H}^{\frac{1}{2}}\left(\Gamma_{c}\right)}=0 \quad \forall \boldsymbol{\psi} \in \boldsymbol{H}^{1}\left(\Omega_{c}\right) .
$$

Since $\boldsymbol{\tau}: \boldsymbol{H}^{1}\left(\Omega_{c}\right) \rightarrow \boldsymbol{H}^{\frac{1}{2}}\left(\Gamma_{c}\right)$ is surjective, it follows that $\boldsymbol{\gamma}_{t}(\xi \boldsymbol{u})=0$.

Lemma A.2. It holds that $D\left(\mathcal{A}_{0}\right)=D\left(\mathcal{A}_{0}^{*}\right)$.

Proof. By definition, we have that

$$
\begin{aligned}
D\left(\mathcal{A}_{0}^{*}\right)=\{ & (\widehat{\boldsymbol{E}}, \widehat{\boldsymbol{H}}) \in \boldsymbol{X} \mid \exists\left(\boldsymbol{F}_{\widehat{\boldsymbol{E}}}, \boldsymbol{F}_{\widehat{\boldsymbol{H}}}\right) \in \boldsymbol{X} \text { s.t. } \\
& \left.\left(\mathcal{A}_{0}(\boldsymbol{E}, \boldsymbol{H}),(\widehat{\boldsymbol{E}}, \widehat{\boldsymbol{H}})\right)_{\boldsymbol{X}}=\left(\left(\boldsymbol{F}_{\widehat{\boldsymbol{E}}}, \boldsymbol{F}_{\widehat{\boldsymbol{H}}}\right),(\boldsymbol{E}, \boldsymbol{H})\right)_{\boldsymbol{X}} \quad \forall(\boldsymbol{E}, \boldsymbol{H}) \in D\left(\mathcal{A}_{0}\right)\right\},
\end{aligned}
$$


where $D\left(\mathcal{A}_{0}\right)=\boldsymbol{H}_{0}($ curl $) \times \boldsymbol{H}($ curl $)$. The inclusion $D\left(\mathcal{A}_{0}\right) \subset D\left(\mathcal{A}_{0}^{*}\right)$ holds due to (3.4). Let us now prove $D\left(\mathcal{A}_{0}^{*}\right) \subset D\left(\mathcal{A}_{0}\right)$. To this aim, let $(\widehat{\boldsymbol{E}}, \widehat{\boldsymbol{H}}) \in D\left(\mathcal{A}_{0}^{*}\right)$. Then, in view of $(\mathrm{A} .3)$ and by the definition of the operator $\mathcal{A}_{0}$ and the scalar product of $\boldsymbol{X}$ (see p. 4), there exists $\left(\boldsymbol{F}_{\widehat{\boldsymbol{E}}}, \boldsymbol{F}_{\widehat{\boldsymbol{H}}}\right) \in \boldsymbol{X}$ such that

$$
\begin{array}{r}
(\operatorname{curl} \boldsymbol{H}, \widehat{\boldsymbol{E}})_{\boldsymbol{L}^{2}(\Omega)}-(\operatorname{curl} \boldsymbol{E}, \widehat{\boldsymbol{H}})_{\boldsymbol{L}^{2}(\Omega)}=\left(\varepsilon \boldsymbol{F}_{\widehat{\boldsymbol{E}}}, \boldsymbol{E}\right)_{\boldsymbol{L}^{2}(\Omega)}+\left(\mu \boldsymbol{F}_{\widehat{\boldsymbol{H}}}, \boldsymbol{H}\right)_{\boldsymbol{L}^{2}(\Omega)} \\
\forall(\boldsymbol{E}, \boldsymbol{H}) \in D\left(\mathcal{A}_{0}\right) .
\end{array}
$$

This leads to

$$
\begin{aligned}
-(\operatorname{curl} \boldsymbol{E}, \widehat{\boldsymbol{H}})_{\boldsymbol{L}^{2}(\Omega)} & =\left(\varepsilon \boldsymbol{F}_{\widehat{\boldsymbol{E}}}, \boldsymbol{E}\right)_{\boldsymbol{L}^{2}(\Omega)} & & \forall \boldsymbol{E} \in \boldsymbol{H}_{0}(\operatorname{curl}) \\
(\operatorname{curl} \boldsymbol{H}, \widehat{\boldsymbol{E}})_{\boldsymbol{L}^{2}(\Omega)} & =\left(\mu \boldsymbol{F}_{\widehat{\boldsymbol{H}}}, \boldsymbol{H}\right)_{\boldsymbol{L}^{2}(\Omega)} & & \forall \boldsymbol{H} \in \boldsymbol{H}(\operatorname{curl}) .
\end{aligned}
$$

Since (A.4) and (A.5) also hold for $\boldsymbol{E}, \boldsymbol{H} \in \mathcal{C}_{0}^{\infty}(\Omega)^{3}$, respectively, we obtain by the definition of the curl operator that $\widehat{\boldsymbol{E}}$ and $\widehat{\boldsymbol{H}}$ lie in $\boldsymbol{H}(\mathbf{c u r l})$ with

$$
\operatorname{curl} \widehat{\boldsymbol{E}}=\mu \boldsymbol{F}_{\widehat{\boldsymbol{H}}} \quad \text { and } \quad \operatorname{curl} \widehat{\boldsymbol{H}}=-\varepsilon \boldsymbol{F}_{\widehat{\boldsymbol{E}}} .
$$

Furthermore, as in the proof of Lemma A.1, we denote the tangential trace operator on $\Gamma$ by $\boldsymbol{\gamma}_{t}: \boldsymbol{H}(\mathbf{c u r l}) \rightarrow$ $\boldsymbol{H}^{-\frac{1}{2}}(\Gamma)$ satisfying

$$
\begin{array}{r}
\left\langle\gamma_{t}(\boldsymbol{u}), \boldsymbol{\tau}(\boldsymbol{\psi})\right\rangle_{\boldsymbol{H}^{-\frac{1}{2}}(\Gamma), \boldsymbol{H}^{\frac{1}{2}}(\Gamma)}=\int_{\Omega} \boldsymbol{u} \cdot \operatorname{curl} \boldsymbol{\psi} \mathrm{d} x-\int_{\Omega} \operatorname{curl} \boldsymbol{u} \cdot \boldsymbol{\psi} \mathrm{d} x \\
\forall \boldsymbol{u} \in \boldsymbol{H}(\operatorname{curl}), \boldsymbol{\psi} \in \boldsymbol{H}^{1}(\Omega),
\end{array}
$$

In view of (A.4), (A.6) and (A.7), we arrive at

$$
\begin{aligned}
\left\langle\gamma_{t}(\widehat{\boldsymbol{E}}), \boldsymbol{\tau}(\boldsymbol{\psi})\right\rangle_{\boldsymbol{H}^{-\frac{1}{2}}(\Gamma), \boldsymbol{H}^{\frac{1}{2}}(\Gamma)} & =(\widehat{\boldsymbol{E}}, \operatorname{curl} \boldsymbol{\psi})_{\boldsymbol{L}^{2}(\Omega)}-(\operatorname{curl} \widehat{\boldsymbol{E}}, \boldsymbol{\psi})_{\boldsymbol{L}^{2}(\Omega)} \\
& =(\widehat{\boldsymbol{E}}, \operatorname{curl} \boldsymbol{\psi})_{\boldsymbol{L}^{2}(\Omega)}-\left(\mu \boldsymbol{F}_{\widehat{\boldsymbol{H}}}, \boldsymbol{\psi}\right)_{\boldsymbol{L}^{2}(\Omega)}=0 \quad \forall \boldsymbol{\psi} \in \boldsymbol{H}^{1}(\Omega),
\end{aligned}
$$

which yields $\gamma_{t}(\widehat{\boldsymbol{E}})=0$. In conclusion, $(\widehat{\boldsymbol{E}}, \widehat{\boldsymbol{H}}) \in D\left(\mathcal{A}_{0}\right)$.

\section{REFERENCES}

[1] A. Alonso and A. Valli, Eddy Current Approximation of Maxwell Equations: Theory, Algorithms and Applications. Springer (2010).

[2] C. Amrouche, C. Bernardi, M. Dauge and V. Girault, Vector potentials in three-dimensional non-smooth domains. Math. Methods Appl. Sci. 21 (1998) 823-864.

[3] F. Brezzi, On the existence, uniqueness and approximation of saddle-point problems arising from Lagrangian multipliers. Rev. Française Automat. Informat. Recherche Opérationnelle Sér. Rouge 8 (1974) 129-151.

[4] P. Ciarlet, Jr. and J. Zou, Fully discrete finite element approaches for time-dependent Maxwell's equations. Numer. Math. 82 (1999) 193-219.

[5] M. Costabel, A remark on the regularity of solutions of Maxwell's equations on Lipschitz domains. Math. Methods Appl. Sci. 12 (1990) 365-368.

[6] M. Costabel and M. Dauge, Singularities of electromagnetic fields in polyhedral domains. Arch. Rational Mech. Anal. 151 $2000221-276$.

[7] M. Costabel, M. Dauge and S. Nicaise, Singularities of Maxwell interface problems. ESAIM: M2AN 33 (1999) 627-649.

[8] P-E. Druet, O. Klein, J. Sprekels, F. Tröltzsch and I.Yousept, Optimal control of three-dimensional state-constrained induction heating problems with nonlocal radiation effects. SIAM J. Control Optim. 49 (2011) 1707-1736.

[9] V. Girault and P. Raviart, Finite Element Methods for Navier-Stokes Equations. Springer-Verlag, Berlin (1986).

[10] M. Hintermüller, K. Ito and K. Kunisch, The primal-dual active set strategy as a semismooth Newton method. SIAM J. Optim. 13 (2003) 865-888. 
[11] M. Hintermüller, A. Laurain I. Yousept, Shape sensitivities for an inverse problem in magnetic induction tomography based on the eddy current model. Inverse Problems 31 (2015) 065006.

[12] R.H.W. Hoppe and I. Yousept, Adaptive edge element approximation of H(curl)-elliptic optimal control problems with control constraints. BIT 55 (2015) 255-277.

[13] M. Kolmbauer and U. Langer, A robust preconditioned minres solver for distributed time-periodic eddy current optimal control problems. SIAM J. Scientific Comput. 34 (2012) B785-B809.

[14] M. Kolmbauer and U. Langer, Efficient solvers for some classes of time-periodic eddy current optimal control problems. In vol. 45 of Numerical Solution of Partial Differential Equations: Theory, Algorithms, and Their Applications, edited by O.P. Iliev, S.D. Margenov, P.D Minev, P.S. Vassilevski and L.T Zikatanov. Springer New York (2013) $203-216$.

[15] J.E. Lagnese and G. Leugering, Time domain decomposition in final value optimal control of the Maxwell system. A tribute to J.L. Lions. ESAIM: COCV 8 (2002) 775-799.

[16] R. Leis, Initial-boundary value problems in mathematical physics. B.G. Teubner, Stuttgart (1986).

[17] A. Logg, K.-A. Mardal, and G.N. Wells, Automated Solution of Differential Equations by the Finite Element Method. Springer, Boston (2012).

[18] P. Monk, An analysis of a mixed method for approximating Maxwell's equations. SIAM J. Numer. Anal. 28 (1991) $1610-1634$.

[19] P. Monk, Finite element methods for Maxwell's equations. Clarendon Press, Oxford (2003).

[20] J.C. Nédélec, Mixed finite elements in $\mathbb{R}^{3}$. Numer. Math. 35 (1980) 315-341.

[21] S. Nicaise, S. Stingelin and F. Tröltzsch, On two optimal control problems for magnetic fields. Comput. Methods Appl. Math. 14 (2014) 555-573.

[22] S. Nicaise, S. Stingelin and F. Tröltzsch, Optimal control of magnetic fields in flow measurement. Discrete Contin. Dyn. Systems 8 (2015) 579-605.

[23] A. Pazy, Semigroups of Linear Operators and Applications to Partial Differential Equations. Springer-Verlag, New York (1983).

[24] R. Picard, On the boundary value problems of electro- and magnetostatics. Proc. Roy. Soc. Edinburgh, Sect. A Math. 92 (1982) 165-174.

[25] F. Tröltzsch, Optimal Control of Partial Differential Equations, Vol. 112 of Grad. Stud. Math. American Mathematical Society, Providence, RI (2010).

[26] F. Tröltzsch and I. Yousept, PDE-constrained optimization of time-dependent 3D electromagnetic induction heating by alternating voltages. ESAIM: M2AN 46 (2012) 709-729.

[27] M. Ulbrich, Semismooth Newton methods for operator equations in function spaces. SIAM J. Optim. 13 (2003) 805-842.

[28] N. Weck, Maxwell's boundary value problem on Riemannian manifolds with nonsmooth boundaries. J. Math. Anal. Appl. 46 (1974) 410-437.

[29] N. Weck, Exact boundary controllability of a Maxwell problem. SIAM J. Control Optim. 38 (2000) 736-750.

[30] I. Yousept, Optimal bilinear control of eddy current equations with grad-div regularization. J. Numer. Math. 23 (2015) 81-98.

[31] I. Yousept, Optimal control of a nonlinear coupled electromagnetic induction heating system with pointwise state constraints. Ann. Acad. Rom. Sci. Ser. Math. Appl. 2 (2010) 45-77.

[32] I. Yousept. Finite element analysis of an optimal control problem in the coefficients of time-harmonic Eddy current equations. J. Optim. Theory Appl. 154 (2012) 879-903.

[33] I. Yousept, Optimal control of Maxwell's equations with regularized state constraints. Comput. Optim. Appl. 52 (2012) 559581.

[34] I. Yousept, Optimal control of quasilinear $\boldsymbol{H}$ (curl)-elliptic partial differential equations in magnetostatic field problems. SIAM J. Control Optim. 51 (2013) 3624-3651. 